\title{
Reduction of an Afterhyperpolarization Current Increases Excitability in Striatal Cholinergic Interneurons in Rat Parkinsonism
}

\author{
Gonzalo Sanchez, Mariano Julian Rodriguez, Pablo Pomata, Lorena Rela, and Mario Gustavo Murer \\ Systems Neuroscience Section, Department of Physiology and Biophysics, School of Medicine, University of Buenos Aires, Buenos Aires C1121ABG, \\ Argentina
}

Striatal cholinergic interneurons show tonic spiking activity in the intact and sliced brain, which stems from intrinsic mechanisms. Because of it, they are also known as "tonically active neurons" (TANs). Another hallmark of TAN electrophysiology is a pause response to appetitive and aversive events and to environmental cues that have predicted these events during learning. Notably, the pause response is lost after the degeneration of dopaminergic neurons in animal models of Parkinson's disease. Moreover, Parkinson's disease patients are in a hypercholinergic state and find some clinical benefit in anticholinergic drugs. Current theories propose that excitatory thalamic inputs conveying information about salient sensory stimuli trigger an intrinsic hyperpolarizing response in the striatal cholinergic interneurons. Moreover, it has been postulated that the loss of the pause response in Parkinson's disease is related to a diminution of $I_{\mathrm{SAHP}}$, a slow outward current that mediates an afterhyperpolarization following a train of action potentials. Here we report that $I_{\mathrm{sAHP}}$ induces a marked spike-frequency adaptation in adult rat striatal cholinergic interneurons, inducing an abrupt end of firing during sustained excitation. Chronic loss of dopaminergic neurons markedly reduces $I_{\mathrm{sAHP}}$ and spike-frequency adaptation in cholinergic interneurons, allowing them to fire continuously and at higher rates during sustained excitation. These findings provide a plausible explanation for the hypercholinergic state in Parkinson's disease. Moreover, a reduction of $I_{\mathrm{SAHP}}$ may alter synchronization of cholinergic interneurons with afferent inputs, thus contributing to the loss of the pause response in Parkinson's disease.

\section{Introduction}

Parkinson's disease is a neurodegenerative disorder caused by the loss of nigrostriatal dopaminergic neurons. Its main symptoms result from a deficient dopaminergic control of striatal mechanisms involved in instrumental learning and action selection and initiation (Albin et al., 1989; Bergman et al., 1998; Schultz, 2007). Acetylcholine released by a small population of interneurons is another main modulator of striatal function (Pisani et al., 2007). Because of their continuous and more or less regular firing activity (Kimura et al., 1984), which in part stems from pacemaker mechanisms (Bennett and Wilson, 1999), cholinergic interneurons are also referred to as "tonically active neurons" (TANs). Another hallmark of TAN electrophysiology is a pause response to rewards and sensory stimuli that induce conditioned behavior

\footnotetext{
Received Dec. 6, 2010; revised Feb. 23, 2011; accepted March 8, 2011.

Author contributions: P.P. and M.G.M. designed research; G.S., M.J.R., and L.R. performed research; G.S., M.J.R., P.P., and L.R. analyzed data; G.S., L.R., and M.G.M. wrote the paper.

This study was supported by Secretaría de Ciencia, Tecnología e Innovación Productiva, Fondo para la Investigación Científica y Tecnológica (Argentina; PICT2007-05-01000, PICT2007-05-0945, PME2003-29), Universidad de Buenos Aires (UBACYT M050), and Consejo Nacional de Investigaciones Científicas y Técnicas (Argentina; PIP0077). We thank Carola Rodriguez for taking care of the animals, Mariela Escande for help with 6-OHDA lesions and behavioral testing, and Eduardo Martín (Universidad de Castilla-La Mancha, España) for his comments on an early version of this manuscript.

Correspondence should be addressed to Gonzalo Sanchez, Systems Neuroscience Section, Department of Physiology and Biophysics, School of Medicine, University of Buenos Aires, 2155 Paraguay Street, Buenos Aires C1121ABG, Argentina. E-mail: neurofis@fmed.uba.ar.

DOI:10.1523/JNEUROSCI.6345-10.2011

Copyright $\odot 2011$ the authors $\quad 0270-6474 / 11 / 316553-12 \$ 15.00 / 0$
}

(Kimura et al., 1984; Apicella et al., 1991), which occurs simultaneously with a burst response in the dopaminergic neurons (Morris et al., 2004). Early models of basal ganglia physiology stressed the existence of a balance between dopamine and acetylcholine in the striatum, where dopamine inhibits cholinergic interneurons. The loss of this balance leads to a "hypercholinergic state" in Parkinson's disease (Barbeau, 1962). In line with this view, the cholinergic interneuron pause response to rewardrelated cues disappears after the degeneration of nigrostriatal dopaminergic neurons (Aosaki et al., 1994), and anticholinergic therapy has played a major role in the treatment of Parkinson's disease before the L-Dopa era (Barbeau, 1962). Previous work in striatal cholinergic interneurons identified a lack of inhibitory control through $\mathrm{D}_{2}$ dopamine receptors (DeBoer et al., 1996; Maurice et al., 2004) and a deficient negative feedback through $\mathrm{M}_{4}$ acetylcholine autoreceptors (Ding et al., 2006) as putative mechanisms underlying a rise in acetylcholine release in Parkinson's disease. However, the mechanism underlying the pause response to reward-related stimuli and its loss after dopaminergic neuron degeneration remains elusive. Current theories propose that an intrinsic hyperpolarizing response triggered by synaptic inputs (Reynolds et al., 2004; Wilson, 2005), alone or in combination with a slowing of pacemaking mechanisms (Maurice et al., 2004; Ding et al., 2006, 2010), underlies the pause. Here we ask whether the excitability of striatal cholinergic interneurons increases in a chronic model of Parkinson's disease, the rat 6-hydroxydopamine (6-OHDA) lesion model, which reproduces 
some of the core behavioral deficits of the illness (Cenci et al., 2002) and has good predictive validity for the antiparkinsonian efficacy of drugs, including anticholinergics (Nakagawa et al., 2004).

\section{Materials and Methods}

Subjects. A total of 91 adult (12-15 weeks old at the time of electrophysiological recordings) male Sprague Dawley rats were used (controls, 12 sham and 40 normal rats, $421 \pm 8$ g; 6-OHDA, $n=39,430 \pm 12$ g). Further experiments were performed in 13 juvenile $(25-35 \mathrm{~d}$ old) rats. Animals were housed five per cage with water and food ad libitum and a 12:12 h light/dark cycle (lights on at 7:00 A.M.) and cared for in accordance with institutional (CICUAL, RS2079/2007, University of Buenos Aires) and government regulations (SENASA, RS617/2002, Argentina).

Lesions. A severe unilateral lesion of mesencephalic dopaminergic neurons was obtained with the neurotoxin 6-OHDA hydrobromide (Sigma) when the rats were 2 months old (190-220 g), as reported previously (Tseng et al., 2001; Zold et al., 2007). Briefly, the rats were treated with desipramine $(25 \mathrm{mg} / \mathrm{kg}$, i.p.) and anesthetized with chloral hydrate $(400 \mathrm{mg} /$ $\mathrm{kg}$, i.p.). With the aid of a stereotaxic instrument (David Kopf Instruments), the neurotoxin (8 $\mu \mathrm{g}$ free base in $4 \mu \mathrm{l}$ of $0.1 \%$ ascorbic acid) was injected at the medial forebrain bundle [stereotaxic coordinates in relation to bregma according to Paxinos and Watson (1997): anterior $-4.3 \mathrm{~mm}$; lateral $1.6 \mathrm{~mm}$; and $8.3 \mathrm{~mm}$ below the cortical surface]. Sham animals received only the ascorbic acid solution. The effective-

ness of the lesion was evaluated with the stepping test and further confirmed postmortem by means of immunohistochemistry as in previous studies (Tseng et al., 2001; Zold et al., 2007). The stepping test was repeated three times between days 20 and 30 after surgery in all the rats. Only those 6-OHDA-lesioned rats that showed less than two adjusting steps with the forelimb contralateral to the lesion during each trial were selected for the experiments. The 6-OHDA lesion was performed 4-8 weeks before the recordings.

Slicing. Rats were anesthetized with chloral hydrate $(400 \mathrm{mg} / \mathrm{kg}$, i.p.) and decapitated. The brain was quickly removed, chilled in ice-cold ACSF (low $\mathrm{Ca}^{2+}$ and high $\mathrm{Mg}^{2+}$ ), and blocked for slicing. The tissue was glued to the tray of a vibratome (Pelco T series 1000, Ted Pella) and $300-\mu \mathrm{m}$-thick coronal slices were cut (approximate distance from bregma $1.50 \mathrm{~mm}$ to $-0.50 \mathrm{~mm}$ ). Slices were submerged in ACSF (low $\mathrm{Ca}^{2+}$ and high $\mathrm{Mg}^{2+}$ ) at $34^{\circ} \mathrm{C}$ for at least $1 \mathrm{~h}$ in a recovery chamber. ACSF composition was as follows (in $\mathrm{mm}$ ): $125 \mathrm{NaCl}, 2.5 \mathrm{KCl}, 1.3$ $\mathrm{NaH}_{2} \mathrm{PO}_{4} \cdot \mathrm{H}_{2} \mathrm{O}, 26 \mathrm{NaHCO}_{3}, 2 \mathrm{CaCl}_{2}, 1 \mathrm{MgCl}_{2}$, and 10 glucose. For low- $\mathrm{Ca}^{2+}$, high- $\mathrm{Mg}^{2+}$ ACSF, $0.5 \mathrm{~mm} \mathrm{CaCl}_{2}$ and $2.5 \mathrm{~mm} \mathrm{MgCl}_{2}$ were used.

Electrophysiological recordings. Slices were transferred to a submersion type chamber perfused by means of a peristaltic pump with ACSF at a constant rate of $2 \mathrm{ml} / \mathrm{min}$; temperature in the recording chamber was set at $34^{\circ} \mathrm{C}$ with a TC-344B temperature controller (Warner Instruments). Cells were sampled from the dorsolateral motor district of the striatum. Visualization was achieved using an upright microscope (Nikon Eclipse) equipped with a $40 \times$ water-immersion objective, DIC optics, and an infrared camera connected to a monitor. Recording electrodes were made with borosilicate capillaries shaped with a puller (P-97, Sutter), filled with internal solution containing the following (in $\mathrm{mM}$ ): $20 \mathrm{KCl}$, 120 K-gluconate, 10 HEPES, $3 \mathrm{Na}_{2} \mathrm{ATP}, 0.3 \mathrm{NaGTP}, 0.1$ EGTA, and 2 $\mathrm{MgCl}_{2}$. Electrode resistance was 4-7 $\mathrm{M} \Omega$ when submerged in ACSF.
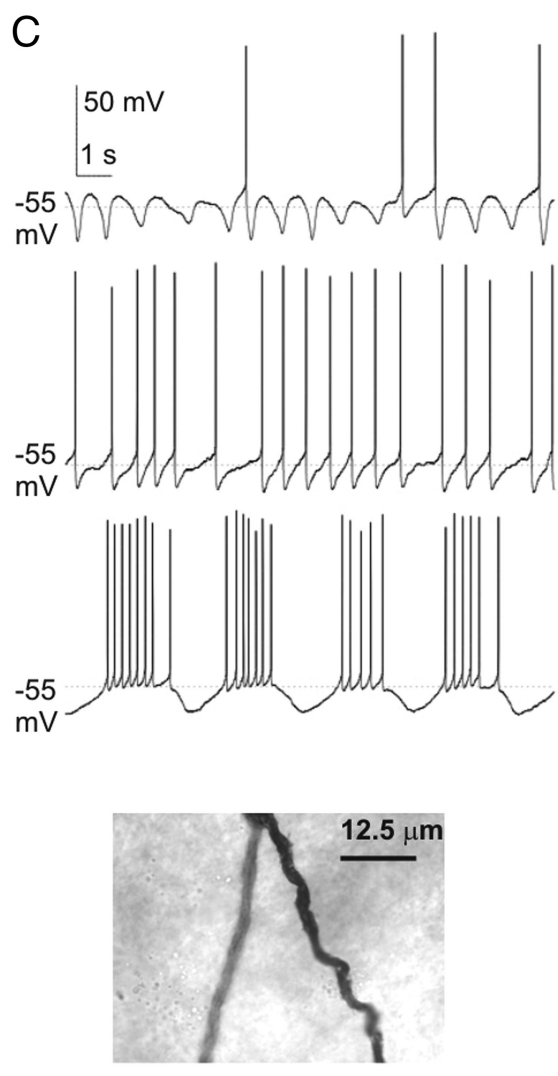
at $20 \mathrm{kHz}$ (Digidata 1322A, Molecular Devices), and acquired on a PC running pClamp 9.2. Cells considered for data analysis fulfilled the following criteria: resting membrane potential between -67 and $-43 \mathrm{mV}$, series resistance between 10 and $50 \mathrm{M} \Omega$, and action potential amplitude $\geq 50 \mathrm{mV}$ from threshold to peak. The liquid junction potential was not compensated. In a few cases, to prevent spontaneous firing or changes in membrane potential due to pharmacological manipulation, minimal DC current was injected to bring cells to the $-60 \mathrm{mV}$ to $-70 \mathrm{mV}$ membrane potential range.

Data analysis. Measurements were performed with ClampFit (pClamp 9.2 software, Axon). GraphPad Prism version 5.00 for Windows (GraphPad Software) was used for data analysis. Statistical differences were established by either two-way repeated-measures ANOVA with Bonferroni post hoc test or two-tailed Student's $t$ test, depending on the number of factors involved. Significance level was set at $p<0.05$, and all data are expressed as mean \pm SEM unless otherwise specified.

Computer simulations. We used a modified and updated version of the striatal cholinergic interneuron model constructed by Maurice et al. (2004). The model is implemented in NEURON (Hines and Carnevale, 1997), and consists of three cylindrical compartments: a $20-\mu \mathrm{m}$-diameter, 20 - $\mu \mathrm{m}$-long soma, connected with two $2-\mu \mathrm{m}$-diameter, $200-\mu \mathrm{m}$-long dendrites. Membrane conductance was $0.1 \mathrm{mS} / \mathrm{cm}^{2}$, capacitance $1 \mu \mathrm{F} / \mathrm{cm}^{2}$, and axial resistivity $70 \Omega / \mathrm{cm}$. Equilibrium voltages for conductances were as follows (in $\mathrm{mV}$ ): -55 passive, $50 \mathrm{Na}^{+},-77 \mathrm{~K}^{+},-20 \mathrm{HCN}$ (mixed cationic current). The model includes the following conductances: fast voltage-dependent and persistent $\mathrm{Na}^{+}$(Maurice et al., 2004), voltage-dependent $\mathrm{K}^{+}\left(\mathrm{Kv} 2, \mathrm{Kv} 4\right.$, and $\left.\mathrm{K}_{\mathrm{IR}}\right)$ (Song et al., 1998; Wilson, 2005), $\mathrm{Ca}^{2+}$-dependent $\mathrm{K}^{+}$(BK, SK, and sAHP) (Bennett et al., 2000; Goldberg et al., 2009) (also see Results), voltage-dependent $\mathrm{Ca}^{2+}$ (L and Q type) (Yan and Surmeier, 1996; Bennett et al., 2000; Goldberg et al., 
A Control
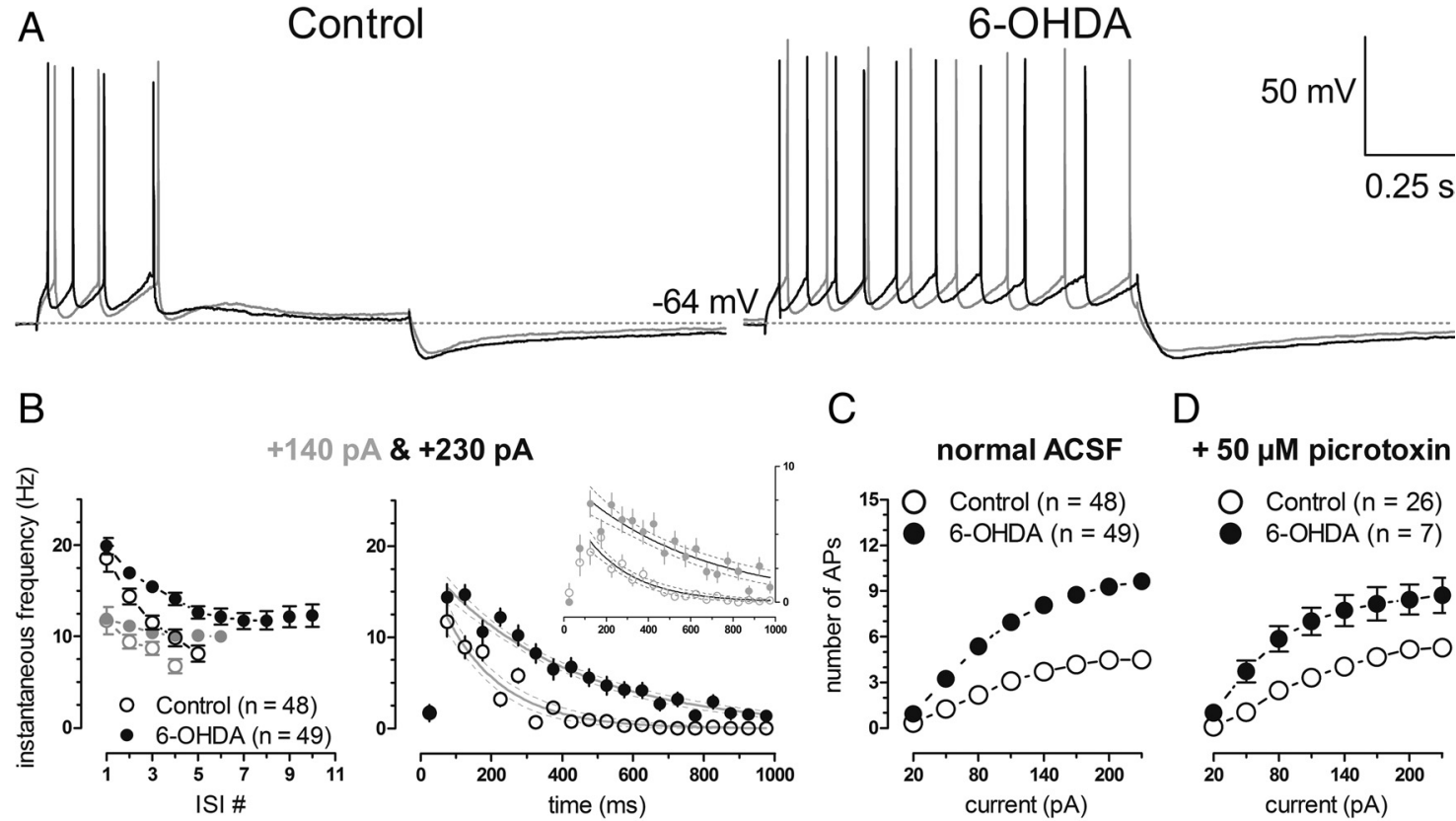

C

$\mathrm{D}$
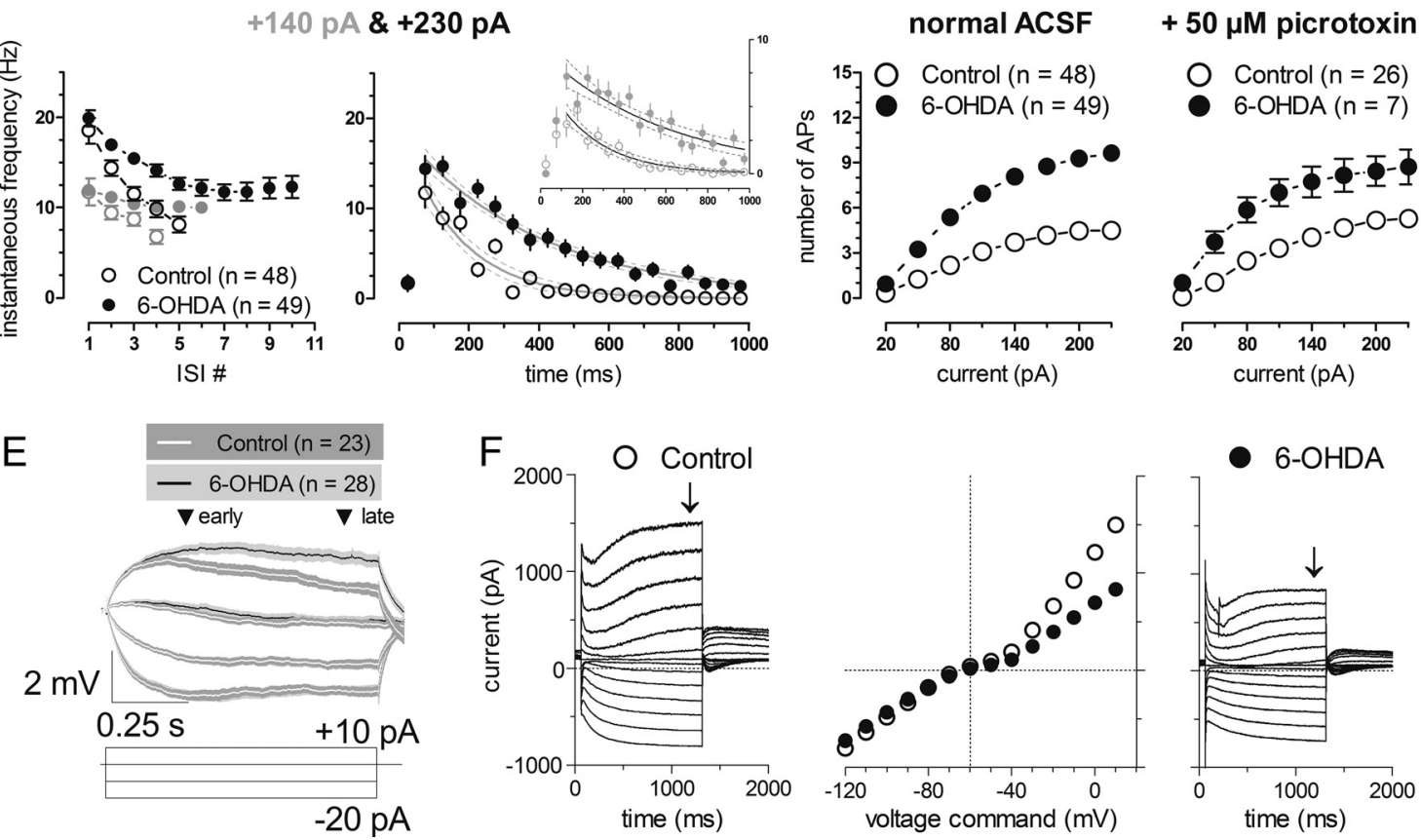

Figure 2. Spike-frequency adaptation is impaired in rats with nigrostriatal lesion. $A$, Representative responses to 140 (gray) and $230 \mathrm{pA}$ (black) $1 \mathrm{~s}$ depolarizing current injection in a control (left) and a 6-OHDA rat (right) striatal cholinergic interneuron. $B$, Instantaneous frequency corresponding to each interspike interval (left) and as a function of time (right, bin size $50 \mathrm{~ms}$ ) during spike trains elicited by $1 \mathrm{~s}$ depolarizing current injection in control and 6-OHDA rats. Right, Separate exponential curves for each group fit the data better than a single curve for the whole dataset (with $p<$ 0.0001 for $230 \mathrm{pA}$ and $p<0.0001$ for $140 \mathrm{pA}$ datasets). Data are mean \pm SEM (circles and error bars) to whom exponentials (curves) were fitted. Dotted lines are the $95 \%$ confidence intervals of the exponential curves. C, Number of spikes fired as a function of current intensity in both experimental groups ( $p<0.0001$ lesion effect, two-way ANOVA). $D$, Effect of picrotoxin in the bath on the number of spikes fired during $1 \mathrm{~s}$ current pulses of different intensities ( $p<0.0001$ lesion effect, two-way ANOVA). In $\boldsymbol{B}-\boldsymbol{D}$, data are mean \pm SEM. $\boldsymbol{E}$, Subthreshold responses of control and 6-OHDA rat interneurons to a series of $1 \mathrm{~s}$ current pulses from -20 to $+10 \mathrm{pA}$ delivered at $10 \mathrm{pA}$ steps (no TTX added; traces are mean $\pm \mathrm{SEM})$. Significant effects of lesion $(p<0.0001)$, time $(p<0.0001$ ), and interaction ( $p=0.016$ ), in a two-way repeated-measures ANOVA ( $p<0.01$, Bonferroni post hoc test for control vs 6-OHDA at "late"). $\boldsymbol{F}$, Representative current responses to a series of voltage commands and I/V plots for a control and a 6-OHDA rat interneuron (holding at $-50 \mathrm{mV}$, steps from -120 to $+10 \mathrm{mV}$ at $\Delta 10 \mathrm{mV}$ for $1 \mathrm{~s}$, measures taken at steady state).

2009), and HCN1 and HCN2 ( $I_{\mathrm{h}}$ currents) (Wilson, 2005). KCNQ conductance $\left(I_{\mathrm{M}}\right.$ current) was not included because $I_{\mathrm{M}}$ blockers had no effect on spike-frequency adaptation in control interneurons (see Results). Intracellular $\mathrm{Ca}^{2+}$ handling was taken from Lazarewicz et al. (2002). It was modified to obtain $\mathrm{Ca}^{2+}$ transients on the order of hundreds of milliseconds in response to single spikes, and a few seconds for spike trains evoked by 230 pA, 1 s current pulses (Bennett et al., 2000). The $\mathrm{Ca}^{2+}$ handling parameters changed were as follows: the dissociation constant of the intracellular Ca-buffer complex, from 0.5 to $1.5 \mathrm{~ms}^{-1}$; the association constant of the calcium pump and extracellular $\mathrm{Ca}^{2+}$, from $5.00 \times$ $10^{-6}$ to $3.75 \times 10^{-6} \mu \mathrm{m}^{3} / \mathrm{s}$; and the total pump density, from 0.2 to 0.6 $\mathrm{mol} / \mathrm{cm}^{2}$. The model of SK conductance was originally from Lazarewicz et al. (2002) and modified according to Bennett et al. (2000), Goldberg and Wilson (2009), and our present results. The model of BK was taken from Khaliq et al. (2003). The model for HCN channels was as adapted by Maurice et al. (2004) from Wang et al. (2001). The models for the other conductances were as implemented by Maurice et al. (2004). The sAHP conductance model was the same as the SK model but with slower kinetics, according to Wilson and Goldberg (2006) and our own results. The ratio slow/fast $\mathrm{Na}^{+}$channel density was 0.4 (Maurice et al., 2004). Max- imal conductance densities of $\mathrm{Na}^{+}$channels were tuned to broadly fit the membrane potential response to current pulses in the soma, and voltageclamp measurements (see Results). Soma densities were as follows (in $\mathrm{S} / \mathrm{cm}^{2}$ ): 0.1 fast $\mathrm{Na}^{+}, 0.04$ slow $\mathrm{Na}^{+}, 7.5 \times 10^{-4} \mathrm{Ca}-\mathrm{L}, 3 \times 10^{-5} \mathrm{Ca}-\mathrm{Q}$, $0.01 \mathrm{Kv} 2,0.05 \mathrm{Kv} 4,0.25 \mathrm{BK}, 0.005 \mathrm{SK}, 0 \mathrm{HCN} 1$, and $0 \mathrm{HCN} 2$. Dendritic densities were as follows: 0.024 fast $\mathrm{Na}^{+}, 0.01$ slow $\mathrm{Na}^{+}, 7.5 \times 10^{-4}$ Ca-L, 0 Ca-Q, $0.01 \mathrm{Kv} 2,0.05 \mathrm{Kv} 4,7 \times 10^{-5} \mathrm{~K}_{\mathrm{IR}}, 0.25 \mathrm{BK}, 0.005 \mathrm{SK}, 9 \times$ $10^{-5} \mathrm{HCN} 1$, and $2.5 \times 10^{-4} \mathrm{HCN} 2$. Model files are available on request.

\section{Results}

Striatal cholinergic interneurons are more excitable in rats with nigrostriatal lesion

In adult rats (12-15 weeks old) with or without nigrostriatal lesion, striatal cholinergic interneurons were recognized thanks to their large cell body and typical electrophysiological properties (Fig. 1): a sag in the membrane potential response elicited by hyperpolarizing current steps, a rebound excitation after current removal, broad action potentials, and distinctive spontaneous firing patterns and subthreshold oscillations (Jiang and North, 
1991; Bennett and Wilson, 1999). Regardless of the lesion, adult striatal cholinergic interneurons show spontaneous activity similar to that previously reported in young rats (Bennett and Wilson, 1999), including regular and irregular firing patterns and rhythmic bursting (Fig. 1C) (average membrane potential \pm SEM when not spiking: $-60.1 \pm 0.5 \mathrm{mV}$ in control rats, $n=63$, $-59.1 \pm 0.6 \mathrm{mV}$ in 6-OHDA rats, $n=62, p=0.2$ ). Overall, 2 of 63 control and 3 of 62 6-OHDA interneurons showed rhythmic bursting. However, chronic dopamine depletion markedly enhances the firing response of cholinergic interneurons to $1 \mathrm{~s}$ steps of depolarizing current (Fig. $2 A-C$ ). In adult controls, cholinergic interneurons show a marked spike-frequency adaptation, and most cells stop firing 300-500 ms after the beginning of current injection ("accommodation"). In contrast, fast adaptation is weak in cholinergic interneurons in 6-OHDA rats, as revealed by exponentials fitted to the instantaneous frequency data shown in Figure $2 B$, showing a slower decay of instantaneous frequency in the 6-OHDA rats (for $230 \mathrm{pA}, 1 \mathrm{~s}$ pulses, tau was 148 $\mathrm{ms}$ in controls and $370 \mathrm{~ms}$ in 6 -OHDA rats, $p<0.0001)$. In addition, cholinergic interneurons in 6-OHDA rats do not show accommodation and thus fire up to two times more spikes than control interneurons during a $1 \mathrm{~s}$ current step (Fig. 2C). Accommodation was not related to GABAergic input (Fig. 2D), but had a correlate in the input resistance of the cells, which gradually decreases after $300 \mathrm{~ms}$ of subthreshold depolarization $(+10 \mathrm{pA})$ in control neurons (from $230 \pm 22$ to $126 \pm 17 \mathrm{M} \Omega, n=23, p<$ 0.05 ) but remains stable in 6-OHDA rats (from $292 \pm 22$ to $249 \pm 28 \mathrm{M} \Omega, n=28$, NS). Thus, input resistance at the end of a +10 pA current pulse was higher in the 6 -OHDA rats $(p<0.01$; Bonferroni post hoc comparisons after significant lesion $\times$ time interaction in a two-way repeated-measures ANOVA) (Fig. 2 E). In good agreement, $I / V$ plots showed smaller current responses to depolarizing voltage steps in 6-OHDA rats (Fig. 2 F), and conductance estimations at around threshold voltages showed a significantly higher conductance in control rats $(16.4 \pm 2.4$ vs $8.9 \pm 1.5$ $\mathrm{pS}$ in 6-OHDA rats, $p=0.028, F$ test).

Notably, striatal cholinergic interneurons of 2-week-old rats with acute dopamine depletion do not show changes in spikefrequency adaptation (Fino et al., 2007). Thus, the use of adult rats that have received a nigrostriatal lesion $4-8$ weeks before the recordings seems to have been important for detecting a defective spike-frequency adaptation in our experiments. In fact, others have noticed spike-frequency adaptation in striatal cholinergic interneurons in adult rats (Jiang and North, 1991; Calabresi et al., 1998), although it is weak or absent in young animals (Bennett et al., 2000). Additional studies in juvenile rats (25-35 d old) confirmed that spike-frequency adaptation is developmentally regulated (Fig. 3).

Spike-frequency adaptation has fast and slow components and is caused by voltage-dependent potassium currents that become active at depolarized potentials and/or calcium-activated potassium currents that mediate afterhyperpolarizations (AHPs) with different time courses (Nicoll, 1988; Storm, 1990). Striatal cholinergic interneurons express several currents that could induce accommodation, including voltage-dependent potassium currents with properties similar to $I_{\mathrm{M}}$ (Song et al., 1998; Bennett et al., 2000), and calcium-dependent potassium currents that mediate a medium (mAHP) and a slow (sAHP) AHP during excitation (SK and $I_{\text {sAHP }}$, respectively) (Goldberg and Wilson, 2005). Barium blocks several potassium currents, including SK, $I_{\mathrm{M}}$, and a hyperpolarization-activated current $\left(\mathrm{K}_{\mathrm{IR}}\right)$ that amplifies sAHP in cholinergic interneurons (Wilson, 2005), but spares others, including some components of $I_{\text {sAHP }}$ (Goldberg et al., 2009) and

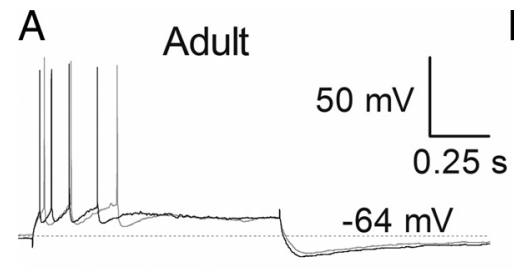

B $\bigcirc$ Adult $(n=48)$
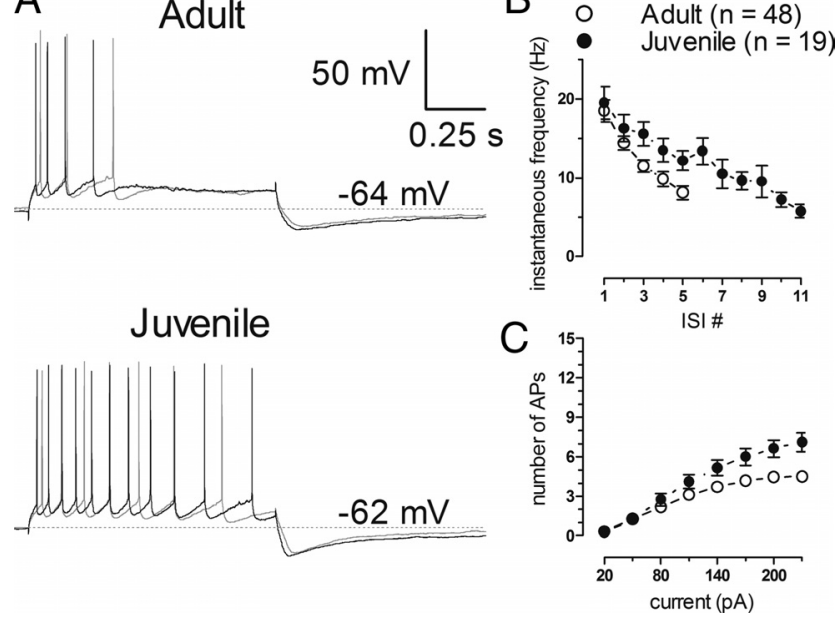

Figure 3. Periadolescent maturation of spike-frequency adaptation in striatal cholinergic interneurons. $\boldsymbol{A}$, Representative responses to 140 (gray) and 230 (black) pA 1 s depolarizing current injection in juvenile ( $25-35 \mathrm{~d}$ old) and adult (12-15 weeks old) rat striatal cholinergic interneurons. $\boldsymbol{B}$, Instantaneous frequency during $230 \mathrm{pA}, 1 \mathrm{~s}$ depolarizing current injection as a function of interspike interval number in juvenile and adult rats ( $p=0.0036$, two exponentials fit best the dataset, F test of best fits). $C$, Number of spikes fired as a function of current intensity in juvenile and adult rats ( $p=0.007$ lesion effect and $p<0.0001$ interaction, two-way ANOVA). Data are mean \pm SEM. Data from adults are the same shown in Figure 2, $B$ and $C$.

$I_{\mathrm{h}}$, another hyperpolarization-activated depolarizing current that shapes the AHPs (Deng et al., 2007; Oswald et al., 2009). Thus, as a first approach toward characterizing the mechanism involved in accommodation, we asked whether barium impairs accommodation in control striatal cholinergic interneurons. Moreover, if barium-sensitive currents mediating accommodation were reduced in 6-OHDA rats, the lesion would occlude the effect of barium on the excitability of striatal cholinergic interneurons.

Bath application of barium $(200 \mu \mathrm{M})$ impaired accommodation in control interneurons, rendering them more responsive to depolarizing current injection, but had no effect on the total number of spikes fired by 6-OHDA rat interneurons (Fig. 4A-C). However, fast spike-frequency adaptation subsists under barium in control and 6-OHDA interneurons (Fig. 4C). Moreover, barium increased input resistance at the end of subthreshold responses to depolarizing current injection in control interneurons (from $60 \pm 17$ to $211 \pm 41 \mathrm{M} \Omega, n=9, p=0.012$ ), but had no effect in 6-OHDA rats (from $187 \pm 52$ to $200 \pm 24 \mathrm{M} \Omega, n=5$, $p=0.85$ ) (Fig. $4 D$ ). Finally, barium did not have noticeable effects on steady-state current evoked by voltage steps in 6-OHDA rats, but markedly increased input resistance in control interneurons, making control $I / V$ plots more similar to those of 6-OHDA rats (Fig. 4E). Indeed, under barium, control and 6-OHDA cholinergic interneurons were almost indistinguishable in terms of subthreshold responses to depolarizing current injection, spike-frequency adaptation, and number of spikes elicited during current steps. Thus, cholinergic interneurons have fast and slow adaptation mechanisms, and the data show a selective disruption of a barium-sensitive mechanism contributing to slow adaptation/accommodation in 6-OHDA rats.

\section{A barium-sensitive component of $I_{\text {sAHP }}$ is reduced in parkinsonism}

Evidence suggests that the pause response of TANs to rewardrelated cues is an intrinsic hyperpolarization triggered by excitatory thalamic input (Matsumoto et al., 2001; Reynolds et al., 2004; Deng et al., 2007; Ding et al., 2010). Currents inducing 

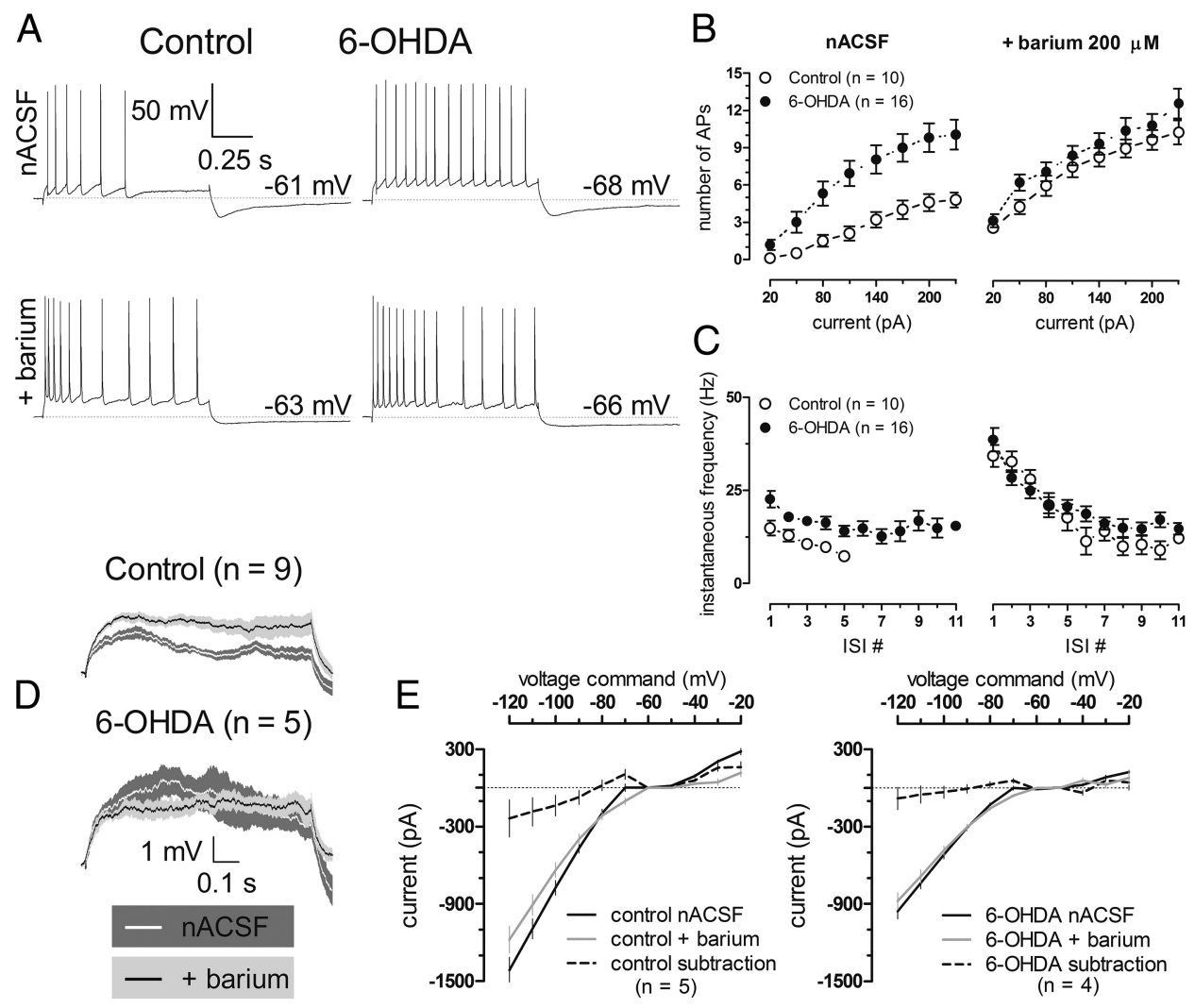

Figure 4. Chronic nigrostriatal degeneration mimics the effect of barium on accommodation and subthreshold responses to depolarizing current injection. $A$, Representative traces showing the effect of bath administration of barium $(200 \mu \mathrm{M})$ on the responses of striatal cholinergic interneurons to $230 \mathrm{pA}, 1 \mathrm{~s}$ current steps, recorded in control and 6-0HDA rats. $\boldsymbol{B}$, Number of spikes fired during $1 \mathrm{~s}$ pulses as a function of current intensity in both experimental groups before and after addition of barium to the bath. Barium had a significant effect in control neurons ( $p<0.0001$, two-way ANOVA) but lacked any effect in 6-OHDA rat interneurons (NS barium effect and interaction, two-way ANOVA). C, Instantaneous frequency corresponding to every interspike interval during spike trains elicited by $230 \mathrm{pA}$, $1 \mathrm{~s}$ current pulses in control and 6-OHDA rats before and after addition of barium (200 $\mu \mathrm{m})$ to the bath. Instantaneous frequency increased significantly in barium compared to normal ACSF (significant effect of barium without group effect and interaction in a two-way ANOVA). Instantaneous frequency did not differ between control interneurons treated with barium and 6-OHDA interneurons with or without barium ( $p=0.88$, Kruskal-Wallis ANOVA comparing plateau instantaneous frequency in control+barium, 6-OHDA, and 6-0HDA + barium). $D$, Subthreshold responses to $1 \mathrm{~s}, 10 \mathrm{pA}$ depolarizing current injection in control and 6-OHDA rat striatal cholinergic interneurons, before and after barium (200 $\mu \mathrm{M})$. Traces are mean $\pm \mathrm{SEM}$ of $n$ neurons per group. $E$, Barium effect in $I / V$ plots of control (left) and 6-OHDA (right) rats (holding potential $-50 \mathrm{mV}$, $1 \mathrm{~s}$ steps from -120 to $-20 \mathrm{mV}$ at $\Delta 10 \mathrm{mV}$, measures taken at steady state). Dashed lines result from the subtraction of data in barium (gray) from data in normal ACSF (black). Barium had no effect on $/ / V$ plots in interneurons of 6-OHDA rats (NS effects of barium and interaction, $p<$ 0.0001 for voltage command), but it markedly reduced the current evoked by voltage commands in control cholinergic interneurons ( $p=0.01$ for barium, $p=0.0026$ for the interaction, $p<$ 0.0001 for voltage command). In $B, C$, and $E$, data are mean \pm SEM.

accommodation are good candidates for that intrinsic mechanism. Among the barium-sensitive currents that can produce slow spike-frequency adaptation are SK, $I_{\mathrm{M}}$, and $I_{\mathrm{sAHP}}$. For instance, classical studies link $I_{\mathrm{M}}$ to spike-frequency adaptation and accommodation in sympathetic ganglia cholinergic neurons (Adams et al., 1982; Wang and McKinnon, 1995) and hippocampal neurons (Madison and Nicoll, 1984).

In striatal cholinergic interneurons, $I_{\mathrm{SK}}$ underlies an $\mathrm{mAHP}$ that follows individual action potentials and regulates interspike intervals (Bennett et al., 2000). Accordingly, interspike intervals are shorter in control striatal cholinergic interneurons under the effect of the specific blocker of SK channels apamin, but accommodation was not affected (Fig. 5A-C). Further suggesting that SK channels do not account for the differences between control and 6-OHDA rats, the peak current and decay constant of the apamin-sensitive current evoked by a simulated action potential (Goldberg and Wilson, 2005 ) were similar in both groups (Fig. $5 D-F$ ). Moreover, although striatal cholinergic interneurons express Kv7/KCNQ channels (Cooper et al., 2001), the specific blocker of $I_{\mathrm{M}} \mathrm{XE}$ 991 lacked any effect on accommodation (Fig. 6).

Another strong candidate to contribute to accommodation (Nicoll, 1988; Storm, 1990) and to the interneuron response to reward-related instructions (Reynolds et al., 2004; Wilson, 2005; Deng et al., 2007) is $I_{\mathrm{sAHP}}$. However, $I_{\mathrm{sAHP}}$ may be insensitive to barium in striatal cholinergic interneurons (Goldberg and Wilson, 2005). We measured $I_{\mathrm{sAHP}}$ using an established protocol (Goldberg and Wilson, 2005) as the tail current elicited after large depolarizing voltage steps (holding at $-50 \mathrm{mV}, 1 \mathrm{~s}$ steps to -40 to $0 \mathrm{mV}, \Delta 10 \mathrm{mV}$, under TTX). Barium $(200 \mu \mathrm{M})$ eliminated an early component that contributes substantially to peak $I_{\mathrm{sAHP}}$, but spared a very long-lasting component (Fig. $7 A, B$ ). Moreover, the number of spikes fired during depolarizing current injection correlated with the peak $I_{\mathrm{sAHP}}$ measured in the same neuron (Fig. $7 C$ ), supporting the view that barium-sensitive $I_{\mathrm{SAHP}}$ contributes to accommodation. Importantly, peak $I_{\mathrm{sAHP}}$ is reduced in rats with chronic nigrostriatal lesion (Fig. 7D).

To be involved in spike-frequency adaptation, $I_{\mathrm{sAHP}}$ should be active at threshold after a few-hundred-millisecond depolarization. Moreover, findings reported by Reynolds et al. (2004) and those summarized in Figures $2 E$ and $4 D$ suggest that $I_{\mathrm{sAHP}}$ may be active at subthreshold potentials. To simulate in voltage clamp the conditions leading to accommodation more closely, striatal cholinergic interneurons were clamped at their resting potential $(-60 \mathrm{mV})$ and stepped for $1 \mathrm{~s}$ under TTX to potentials similar to 
those seen during current injection in current-clamp $(-50 \mathrm{mV}$ to $-38 \mathrm{mV}, \Delta 2$ $\mathrm{mV}$ ). In this condition, a slow bariumsensitive outward current increased in amplitude along the steps (Fig. 7E). Importantly, the current outlasted the voltage steps, giving rise to an almost pure "barium-sensitive $I_{\text {sAHP }}$ " (Fig. $7 F$ ).

Recent studies show that different voltage-dependent calcium currents are coupled to $I_{\mathrm{BK}}, I_{\mathrm{SK}}$, and $I_{\mathrm{sAHP}}$ in striatal cholinergic interneurons, with dihydropyridinesensitive L-type calcium channels being selectively coupled to $I_{\text {sAHP }}$ (Goldberg and Wilson, 2005; Goldberg et al., 2009). However, neither nifedipine nor nimodipine impaired accommodation in striatal interneurons from normal adult rats (Fig. 8). Moreover, addition of barium could still impair accommodation under nifedipine, suggesting that accommodation is independent of L-type calcium channeldependent potassium currents.

\section{A model interneuron with reduced $I_{\text {sAHP }}$ behaves similarly to striatal cholinergic interneurons after dopamine depletion}

Simulations in an updated computational model of the striatal cholinergic interneuron (Maurice et al., 2004) strengthen the view that $I_{\text {sAHP }}$ contributes to accommodation (Fig. 9). The three-compartment model neuron with fast and persistent sodium currents, voltage- and calciumdependent potassium currents $\left(\mathrm{K}_{\mathrm{IR}}, \mathrm{Kv} 2\right.$, $\mathrm{Kv} 4, \mathrm{BK}, \mathrm{SK}$, and $\left.I_{\mathrm{sAHP}}\right)$, L- and Q-type voltage-dependent calcium currents, and $I_{\mathrm{h}}$ captured the basic electrophysiological properties of striatal cholinergic interneurons, including the marked accommodation seen during depolarizing current injection (Fig. 9A). Reducing $I_{\text {sAHP }}$ in the model neuron impairs accommodation (Fig. $9 A, E$ ) and produces changes in $I / V$ plots that mimic very closely the effect of the 6-OHDA lesion. $I_{\text {sAHP }}$ is active at a holding potential of $-50 \mathrm{mV}$ both in control interneurons and the model neuron (Fig. 9B, C), and the 6-OHDA lesion reduces the current that is measured at this holding potential (Fig. $9 B, C)$. Moreover, depolarizing steps induce smaller slow outward currents in 6-OHDA rats and in the model neuron lacking $I_{\text {sAHP }}$ (Fig. 9B,D). Importantly, the model predicted that hyperpolarizing voltage steps from a holding potential of $-50 \mathrm{mV}$ would evoke reduced currents in 6-OHDA rats (Fig. 9C; see also Fig. $4 E$ ). As $I_{\text {sAHP }}$ is active at $-50 \mathrm{mV}$, and because of its slow decaying time, it makes a substantial contribution to the current measured at the end of $1 \mathrm{~s}$ steps when the membrane potential is below the potassium equilibrium potential (e.g., $-150 \mathrm{mV}$ ) (Fig. $9 C)$. Thus, the current measured at potentials below $E_{\mathrm{K}^{+}}$is reduced in 6-OHDA rats and the model neuron lacking $I_{\text {sAHP }}$ (and also in control rats treated with barium, which is not a new finding, except that this barium effect is usually attributed to the blockade of $\mathrm{K}_{\mathrm{ir}}$ and other potassium currents).

In sum, the data show that a depolarization-activated bariumsensitive current that operates at around-threshold membrane potentials exerts a powerful control of excitability in striatal cho-

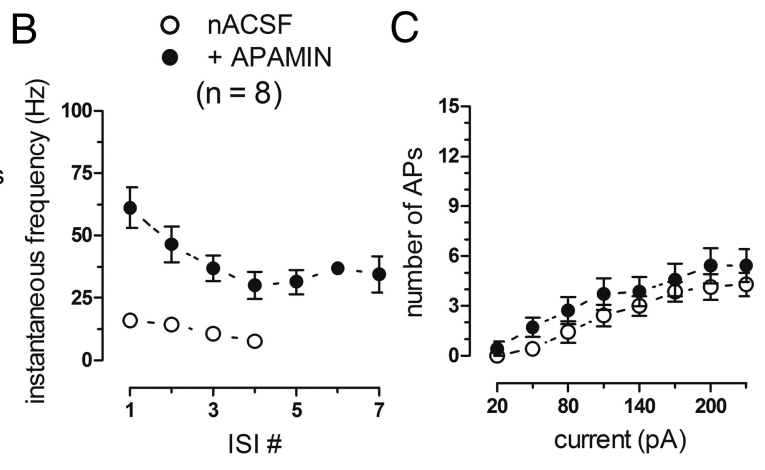

$-66 \mathrm{mV}$
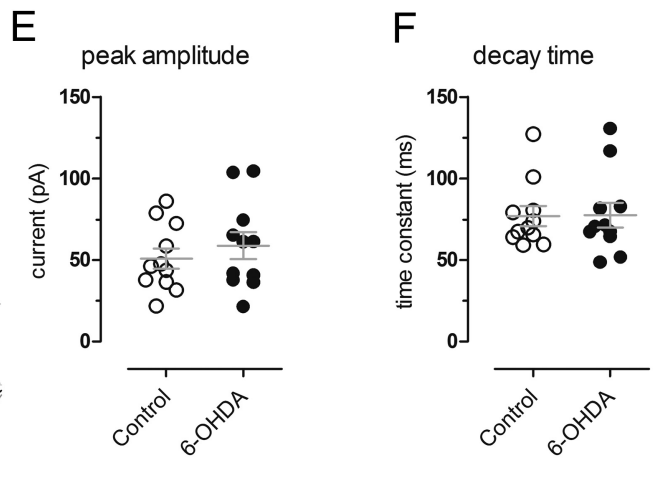

$-62 \mathrm{mV}$

Figure 5. SK current regulates interspike intervals but has no influence on accommodation. $A$, Representative example of the effect of

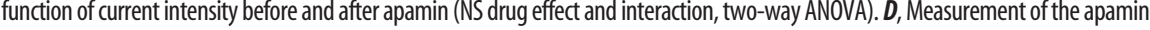
sitive current in a representative voltage-clamp experiment (holding at $-62 \mathrm{mV}$, step to $0 \mathrm{mV}$ for $2.3 \mathrm{~ms}$ ). $\boldsymbol{E}$, $\boldsymbol{F}$, Individual measures of SK current peak amplitude ( $\boldsymbol{E}, p=0.45$, unpaired $t$ test) and decay time $(\boldsymbol{F}, p=0.84$, Mann-Whitney test) in control (open symbols) and 6-OHDA (black symbols) rats. The horizontal lines are the mean and the error bars the SEM.
A
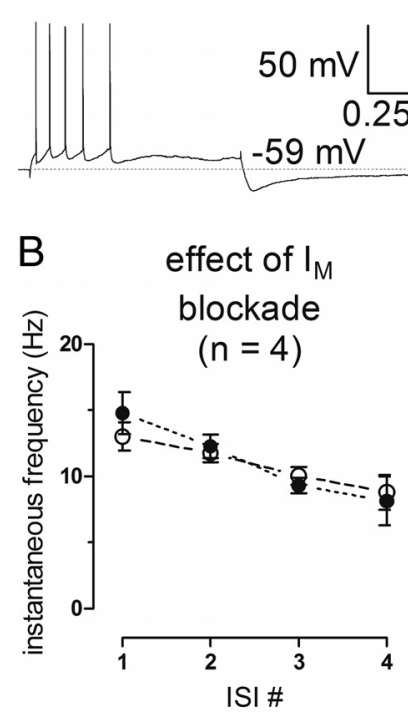

$+\mathrm{XE991}$
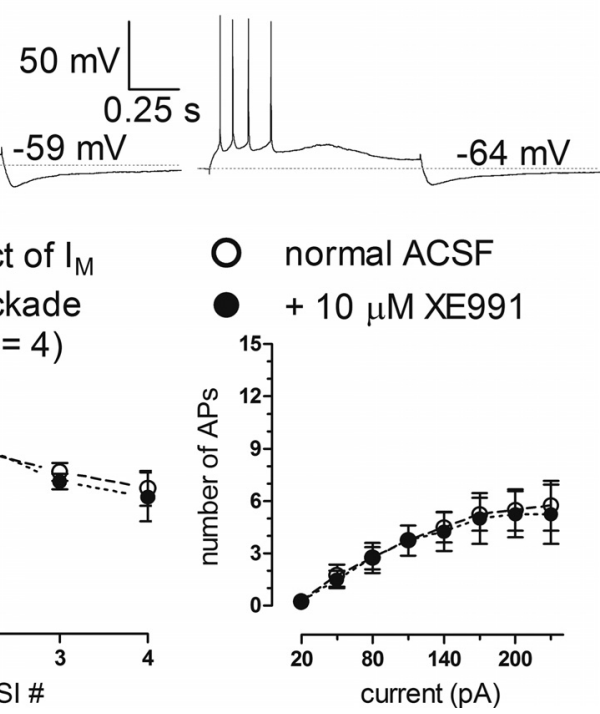

Figure 6. Lack of effect of $I_{M}$ blockers on accommodation. $A$, Representative response to 230 $\mathrm{pA}$, $1 \mathrm{~s}$ depolarizing current injection in an adult rat striatal cholinergic interneuron in ACSF and ACSF with XE-991 (10 $\mu \mathrm{M})$. $\boldsymbol{B}$, Instantaneous frequency corresponding to every interspike interval during a $230 \mathrm{pA}, 1 \mathrm{~s}$ current pulse (left) and number of spikes during the pulse versus current intensity (right) in control rats before (open symbols) and after (black symbols) addition of XE-991 (10 $\mu \mathrm{M}, n=3)$ or linopirdine (10 $\mu \mathrm{m}, n=1)$ to the bath (data pooled for statistical analysis, NS lesion effect and interaction, two-way ANOVAs, data are mean \pm SEM). 

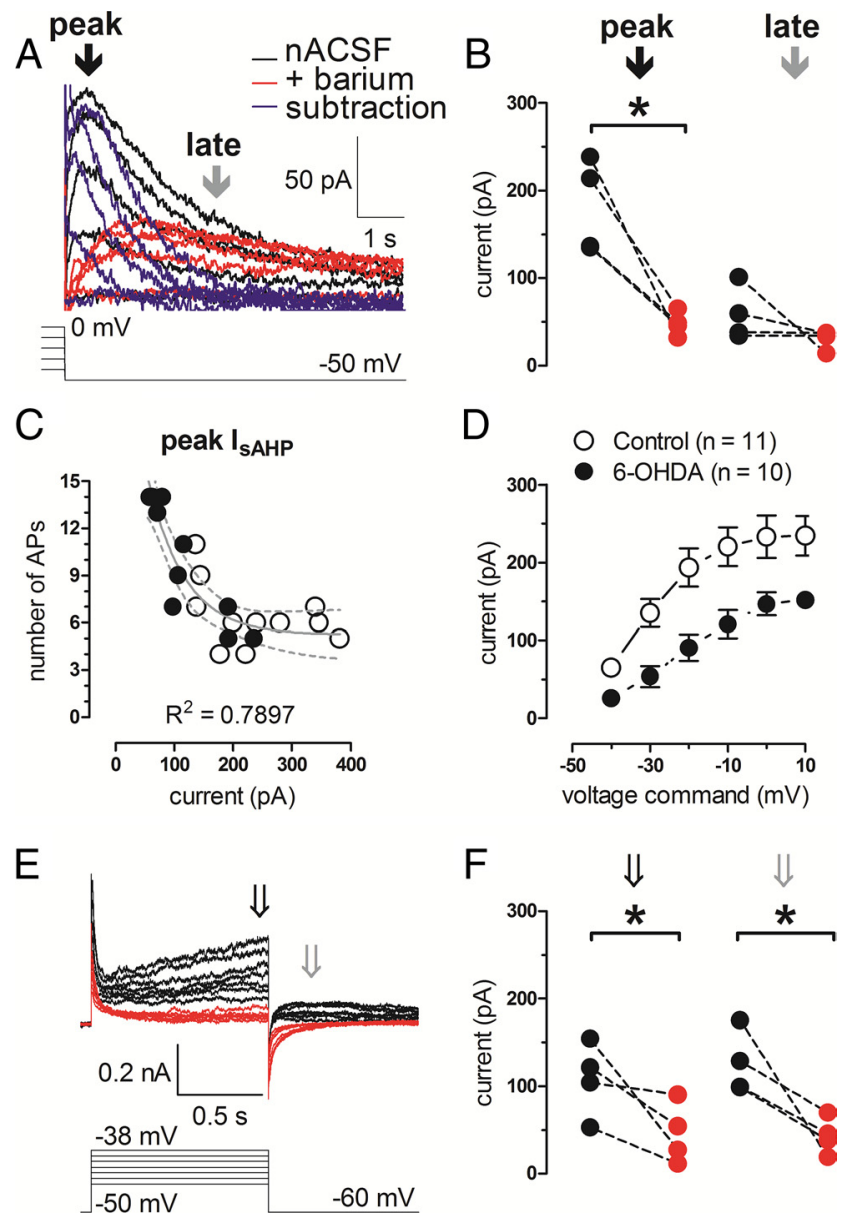

Figure 7. $I_{\text {SAHP }}$ is reduced in rats with chronic nigrostriatal lesion. $A$, An established voltageclamp protocol (holding at $-50 \mathrm{mV}$, $1 \mathrm{~s}$ step from -40 to $0 \mathrm{mV}, \Delta 10 \mathrm{mV}$ ) reveals a marked $I_{\text {sAHP }}$ (black) in a representative striatal cholinergic interneuron. Addition of $200 \mu \mathrm{m}$ barium (red) substantially reduced the peak current. The barium-sensitive current is shown in blue. $\boldsymbol{B}$, Effect of barium $(200 \mu \mathrm{M})$ on $I_{\text {SAHP }}$ at the time of the peak current (black arrow in $A, p=0.018$, paired $t$ test) and $5 \mathrm{~s}$ after the end of the step command (gray arrow in $A, p=0.27$ ) in four control cholinergic interneurons. $C$, Correlation between the number of action potentials delivered by cholinergic neurons during $1 \mathrm{~s}, 230 \mathrm{pA}$ current pulses and the peak $I_{\text {sAPP }}$ measured after a step to $10 \mathrm{mV}$ from a holding potential of $-50 \mathrm{mV}$ in the same neurons. Each circle is a neuron (white are controls, $n=11$, black are 6-OHDA, $n=10$ ). $\boldsymbol{D}$, Peak $I_{\text {sAHP }}$ evoked by different voltage commands in control and 6-OHDA rats ( $p=0.002$, significant lesion effect, two-way ANOVA; same data as in $\boldsymbol{C}$. Data are mean \pm SEM. $\boldsymbol{E}$, Representative example of the slow outward current evoked by small amplitude depolarizing voltage commands in a control neuron before (black) and under barium (red). $\boldsymbol{F}$, Current amplitude of the current evoked by the voltage-clamp protocol shown in $\boldsymbol{E}$ in normal ACSF (black symbols) and after the addition of barium to the bath (red symbols). The current was measured at the end of the voltage command (black arrow in $E, p=0.041$ ) and as a tail current at its peak (gray arrow in $E, p=0.044$, paired $t$ tests).

linergic interneurons. Moreover, a reduction of this current may account for the lack of accommodation seen in rats with nigrostriatal lesion. Barium-insensitive components of $I_{\text {sAHP }}$ may be called into play when cholinergic interneurons fire at high rates and may be sufficient for the generation of spontaneous burst firing as suggested previously (Goldberg and Wilson, 2005) (Fig. 1D).

\section{Reduced mAHP with normal SK current after nigrostriatal lesion}

To study in more detail the effect of the lesion and barium on action potential properties, we analyzed phase-plane plots showing the rate of change in the membrane potential $(\mathrm{d} V / \mathrm{d} t)$ against
A
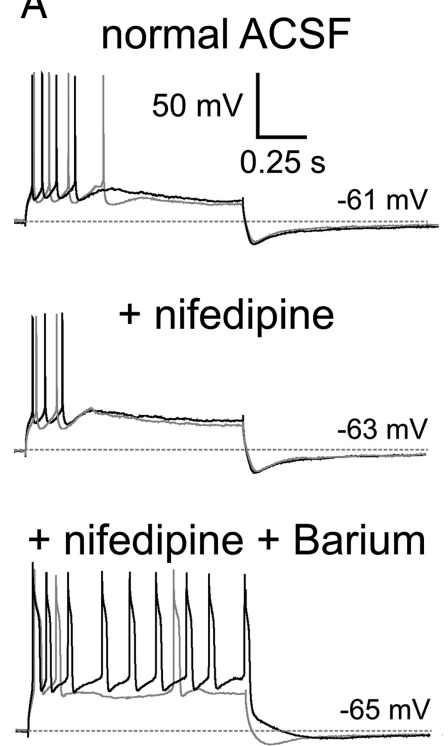

B
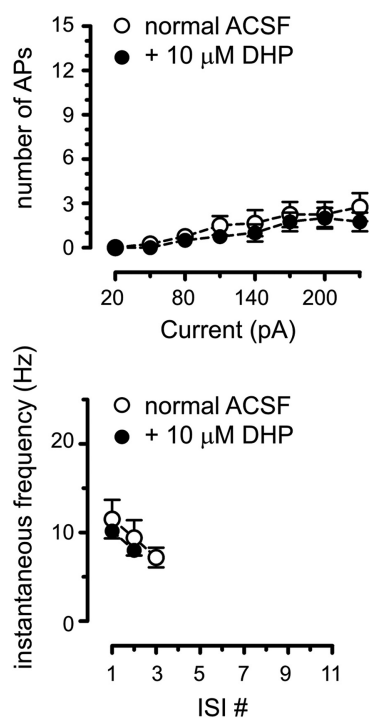

Figure 8. Dihydropyridines do not impair accommodation in adult striatal cholinergic interneurons. A, Representative responses of adult rat cholinergic interneurons to 230 (gray) and 320 (black) pA 1 s depolarizing current injection in normal ACSF (top), during perfusion with $10 \mu \mathrm{m}$ nifedipine (middle) and during perfusion with $10 \mu \mathrm{m}$ nifedipine and $200 \mu \mathrm{m}$ barium (bottom). Washout of barium with sequential application of 10 and $20 \mu \mathrm{m}$ nifedipine restored the accommodation observed in normal ACSF ( $n=1$, not shown). $\boldsymbol{B}$, Quantification of the number of spikes during the pulse (top) and the progression of instantaneous frequency along the 230 pA pulse (bottom) in normal ACSF (open symbols) and in the presence of dihydropyridines (black symbols; $n=4$, pooled data from 2 interneurons treated with $10 \mu \mathrm{m}$ nimodipine and 2 interneurons treated with $10 \mu \mathrm{m}$ nifedipine; two-way ANOVA rendered a significant effect of current intensity, $p=0.0004$ and NS effects of the drug nor interaction). Data are mean \pm SEM.

the instantaneous membrane potential (Bean, 2007). In phaseplane plots, action potentials of cholinergic interneurons look like orbits starting with a marked change of $\mathrm{d} V / \mathrm{d} t$ (threshold). The first orbit in a spike train looks similar in control and 6-OHDA interneurons (Fig. 10A). The following orbits show a slower slope in the rising phase, suggesting that interspike intervals may not allow a complete recovery of the action current (Fig. $10 \mathrm{~A})$. Moreover, an inflection in the repolarizing phase of the action potential becomes more marked during the train, probably because of the inactivation of BK calcium-activated potassium channels, which are known to contribute to action potential repolarization in cholinergic interneurons (Bennett et al., 2000; Goldberg and Wilson, 2005). Quantitative comparisons between action potentials from control and 6-OHDA rat interneurons are provided in Table 1. The lesion does not affect action potential width, which depends on $I_{\mathrm{BK}}$. However, it has an effect on $\mathrm{mAHP}$ amplitude, despite the fact that $I_{\mathrm{SK}}$ is not affected by the lesion (Fig. 5). This lesion effect is more marked in spikes occurring at the time at which accommodation takes place in control neurons (Table 1), suggesting a contribution of $I_{\mathrm{sAHP}}$ to the mAHP. An unexpected finding was that the first action potential in the train is of higher amplitude in 6-OHDA rats. Finally, the model interneuron with reduced $I_{\text {sAHP }}$ shows a $30 \%$ smaller mAHP with normal action potentials. Thus, the lesion may induce subtle action potential changes that are not related to a reduction of $I_{\mathrm{sAHP}}$; nevertheless, a reduction of $I_{\mathrm{sAHP}}$ stands as the more plausible explanation for the lesion-induced changes in spike-frequency adaptation. 
A Control

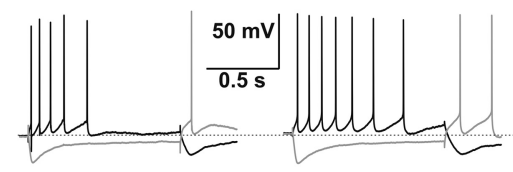

B

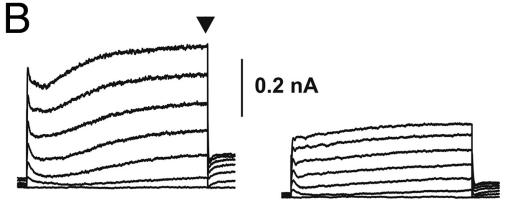

C

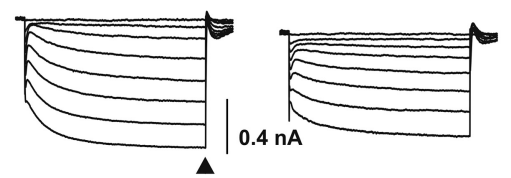

D

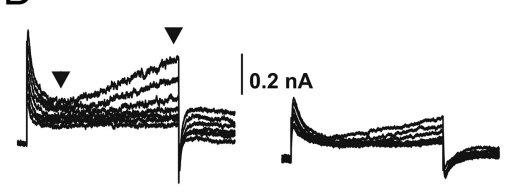

-60
$m V$

$\circ$ control $(n=12)$
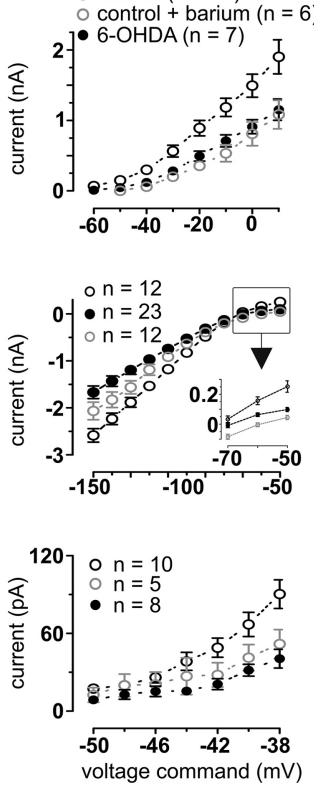

“Control”
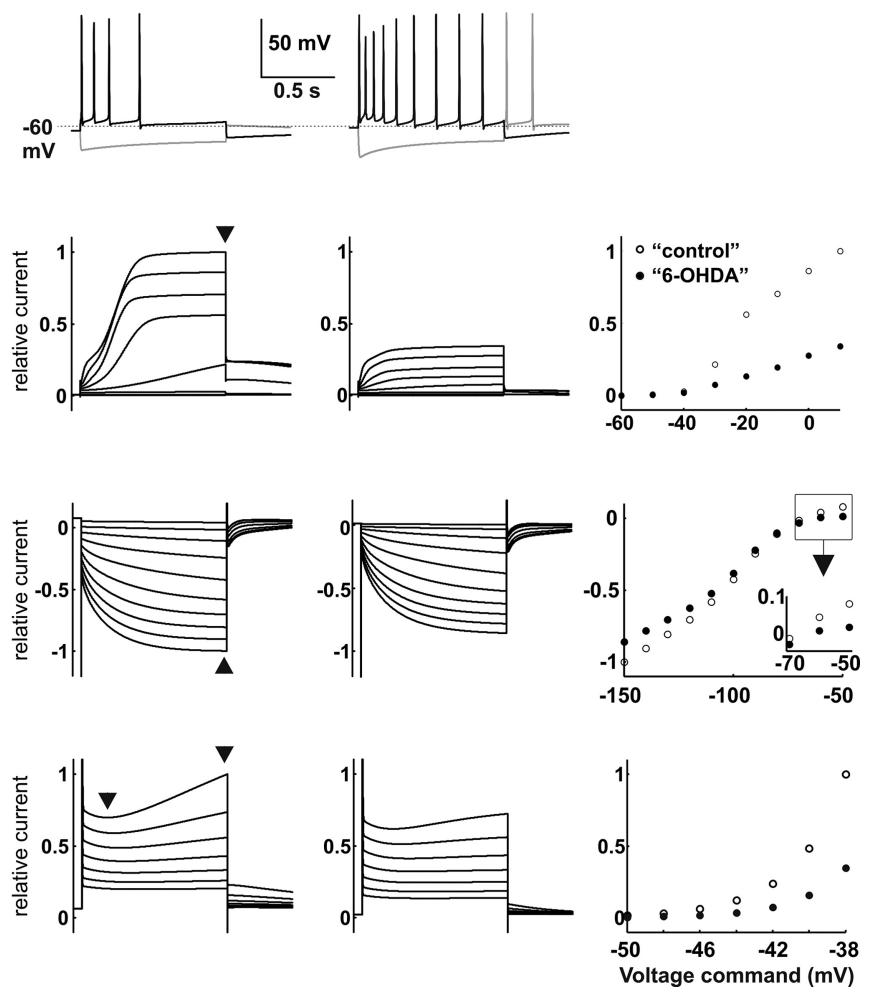

Figure 9. A model striatal cholinergic interneuron lacking $I_{\text {sAHP }}$ shows impaired accommodation and voltage-clamp responses similar to 6-0HDA rat interneurons. $A$, Responses of representative

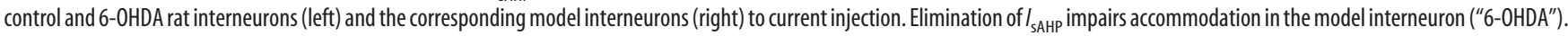
Current pulses: $1 \mathrm{~s},-250 \mathrm{pA}$ (gray) and $230 \mathrm{pA}$ (black). $\boldsymbol{B}-\boldsymbol{D}$, Comparisons between real and simulated voltage-clamp experiments, with representative traces and $I / V$ plots. $\boldsymbol{B}$, Response of striatal cholinergic interneurons (left) and the model interneuron (right) to $1 \mathrm{~s} \mathrm{depolarizing} \mathrm{voltage} \mathrm{steps} \mathrm{from} \mathrm{a} \mathrm{holding} \mathrm{potential} \mathrm{of}-50 \mathrm{mV}(\Delta 10 \mathrm{mV}$, measures taken at arrowhead). Note the reduced outward currents in interneurons recorded from 6-OHDA rats and the model interneuron lacking $I_{\text {sAHP, }}$ and the effect of barium on $I / V$ plots from control cholinergic interneurons. $C$, Responses to hyperpolarizing voltage steps (holding at $-50 \mathrm{mV}, \Delta 10 \mathrm{mV}$, measures taken at arrowhead, see text for details). D, Voltage-clamp protocol designed to study in more detail the slow current activated by around-threshold voltage steps $(-50 \mathrm{mV}$ to $-38 \mathrm{mV}, \Delta 2 \mathrm{mV})$ from a holding potential that is closer to the subthreshold membrane potential of cholinergic interneurons ( $-60 \mathrm{mV})$. Measures for the $I / V$ plots are the difference between arrowheads. Data in the $I / V$ plots from recorded interneurons are mean \pm SEM of $n$ neurons.

\section{Nigrostriatal lesion delays transition from repetitive spiking to silence during prolonged excitation}

Striatal cholinergic interneurons fire tonically in vivo and can show pacemaker-like activity in slices (Bennett and Wilson, 1999) (Fig. 1), suggesting that intrinsic mechanisms allow them to buffer synaptic influences and fire at a preferred frequency. Repetitive spiking results from an interplay between inward and outward currents that are faster than $I_{\mathrm{sAHP}}$ (Bennett et al., 2000; Maurice et al., 2004). However, as the slow outward current described here is active at threshold, it seemed very likely that repetitive spiking would change after chronic dopamine depletion. One method to investigate oscillatory behavior in neurons is by forcing transitions between quiescent and repetitive spiking states by smoothly increasing or decreasing injected current (Izhikevich, 2007). We forced cholinergic interneurons into a quiescent state with hyperpolarizing current and then delivered a slowly depolarizing ramp followed by a symmetric hyperpolarizing ramp (Fig. 11 A). In the transition from quiescence to spiking, the minimal current needed to induce firing (threshold) was similar in interneurons of control ( $36 \pm 10 \mathrm{pA}, n=28 ;-41.9 \pm 0.6$ $\mathrm{mV})$ and 6 -OHDA $(30 \pm 13 \mathrm{pA}, n=12 ;-41.9 \pm 2.4 \mathrm{mV})$ rats. After the first spike, instead of increasing as depolarizing current increased, firing rate reached a preferred frequency in both experimental groups, but the preferred frequency in 6-OHDA rats $(22.9 \pm 1.5 \mathrm{~Hz})$ doubled that in controls $(11.4 \pm 0.7 \mathrm{~Hz}$, unpaired $t$ test, $p<0.00001$ ) (Fig. $11 B, C$ ). Eventually, all interneurons suffered a transition from repetitive spiking to quiescence during the depolarizing ramp or after slope reversal. Note that this transition takes place despite substantially more depolarizing current flowing into the cell body ( $841 \pm 33 \mathrm{pA}$ in controls, $750 \pm 58 \mathrm{pA}$ in 6-OHDA rats) than in the transition from quiescence to spiking (see above, threshold). Importantly, control interneurons stopped firing early during the depolarizing ramp or shortly after slope reversal (median: 3.48 s, 95\% confidence interval: $3.38-$ $3.57 \mathrm{~s}$ ), whereas 6-OHDA rat interneurons could still spike long after current started to ramp down (median: $3.76 \mathrm{~s}, 95 \%$ confidence interval: $3.48-4.08 s ; p=0.013$, Kruskal-Wallis ANOVA). Note also that the switch from repetitive spiking to quiescence in control interneurons may take place abruptly, without a preceding increase in firing rate, or be preceded by a few short interspike intervals (Fig. 11A), resembling transitions from tonic firing to pauses as seen in TANs in relation to reward-related stimuli (Kimura et al., 1984; Apicella et al., 1991; Aosaki et al., 1994; Matsumoto et al., 2001; Blazquez et al., 2002; Ravel et al., 2003; Morris et al., 2004).

Thus, the data strongly argue that striatal cholinergic interneurons show sharp transitions from quiescence to repetitive spiking and from repetitive spiking to quiescence, where the latter quiescent state tolerates strong somatic excitation (Prescott et al., 2006; Izhikevich, 2007; Prescott and Sejnowski, 2008). In rats with chronic nigrostriatal lesion, the transition to repetitive spiking is normal, but the preferred firing frequency is two times 
A a $\mathrm{b} c$
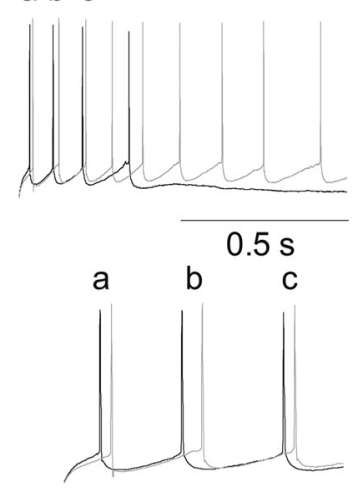

$5 \overline{0 \mathrm{~ms}}$
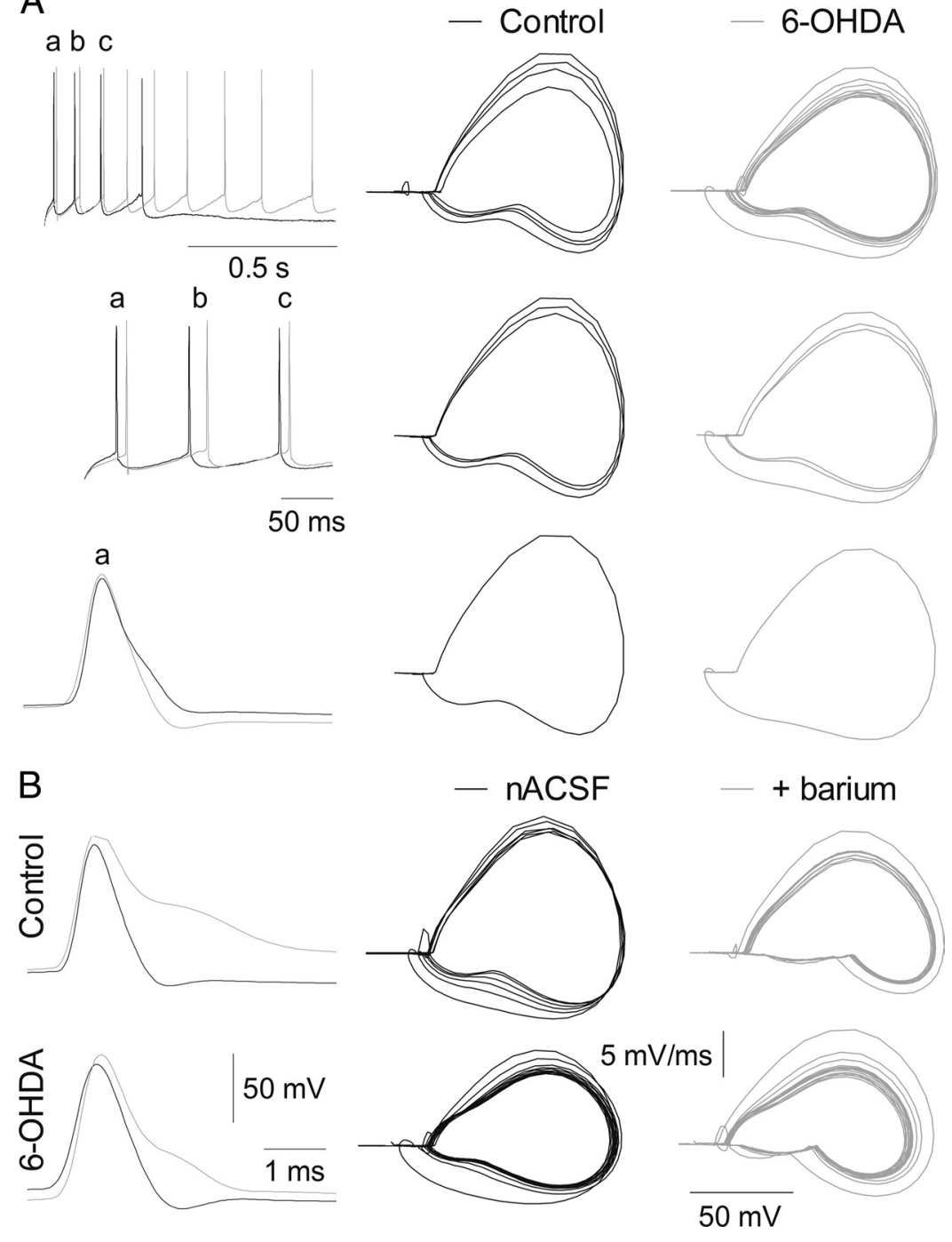

Figure 10. Normal action potential orbits in 6-0HDA rats. $\boldsymbol{A}$, The left column shows the spike trains depicted in Figure $2 A$ in successively expanded timescales (from top to bottom). The two right columns show phase-plane analysis of the spikes shown at the left. Note that the rising and falling phases of action potentials become slower during spike trains in control (middle) and 6-OHDA (right) rat interneurons. $B$, The left column shows in an expanded timescale the first spike of each spike train shown in Figure $4 A$, which correspond to interneurons recorded in a control (top) and a 6-0HDA (bottom) rat, without (black traces) and with (gray traces) barium in the bath. The two right columns show phase-plane analysis of the spike trains shown in Figure $4 A$ in normal ACSF (left) and in the presence of barium (right). See Table 1 for quantitative comparisons.

higher than in controls, and repetitive spiking is sustained for longer during excitation, probably because of the reduced moderating action of $I_{\text {sAHP. }}$.

\section{Discussion}

Striatal cholinergic interneurons undergo a dramatic increase in excitability in chronic dopamine deficiency, which translates into reduced spike-frequency adaptation and a twofold increase in preferred frequency of repetitive spiking. These changes are related to a diminution of a slow depolarization-activated outward current that contributes substantially to $I_{\text {sAHP. }}$.

$I_{\text {sAHP }}$ is related to a variety of computational properties of neurons, including spike-frequency adaptation (Adams et al., 1982; Madison and Nicoll, 1984), gain of input-to-output transformations (Zhang and Arsenault, 2005; Thurley et al., 2008) and preference for spike-time or spike-rate coding (Prescott et al., 2006; Prescott and Sejnowski, 2008). Despite its importance, its molecular correlates remain unidentified (Stocker, 2004). Indeed, $I_{\text {sAHP }}$ may be related to different calcium and voltagedependent molecular entities in different neurons (Faber and Sah, 2005; Tzingounis and Nicoll, 2008). In striatal cholinergic interneurons, $I_{\text {sAHP }}$ is often perceived as a barium-insensitive current activated by calcium load during long subthreshold depolarizations and spike trains (Goldberg and Wilson, 2005; Goldberg et al., 2009). Published findings gave hints about the existence of different slow outward currents in striatal cholinergic interneurons (Song et al., 1998; Bennett et al., 2000; Goldberg and Wilson, 2005); however, our dissection of $I_{\text {sAHP }}$ into two depolarization-activated components based on barium sensitivity is novel. Our results indicate that the barium-sensitive component is primarily responsible for accommodation and that its reduction accounts for the poor spikefrequency adaptation seen in rats with chronic nigrostriatal lesion.

Based on current literature, a reduced sensitivity of $I_{\text {sAHP }}$ to calcium or a reduction of the calcium available to activate $I_{\text {sAHP }}$ could explain the increased excitability in the parkinsonian condition. An intriguing finding of the present study is that blocking L-type voltage-dependent calcium channels with dihydropyridines does not impair accommodation. However, $I_{\text {sAHP }}$ is selectively coupled to L-type dihydropyridine-sensitive calcium channels in striatal cholinergic interneurons (Goldberg and Wilson, 2005; Goldberg et al., 2009). Moreover, $I_{\mathrm{BK}}$ (action potential half-width did not change) and $I_{\mathrm{SK}}$ (apamin-sensitive current did not change), and presumptively their calcium regulation through Q-type and N-type voltagedependent calcium channels (Goldberg and Wilson, 2005), were not altered by the lesion. Thus, the current we call here barium-sensitive $I_{\text {sAHP }}$ may be coupled to calcium sources through an unrecognized mechanism or be an uncommon $I_{\mathrm{M}}$-like current lacking sensitivity to typical $I_{\mathrm{M}}$ blockers (Tzingounis and Nicoll, 2008). Therefore, it may be different from the dihydropyridine-sensitive barium-insensitive $I_{\text {sAHP }}$ component that mediates slow bursting in cholinergic interneurons (Figs. 1C, 7A) (Goldberg and Wilson, 2005). Further studies are needed to unveil the molecular mechanisms leading to $I_{\mathrm{sAHP}}$ reduction in parkinsonism.

Spike-frequency adaptation is defined as a decrease in firing rate during sustained current injection, but the underlying mechanisms have far-reaching consequences on the function of neurons (Izhikevich, 2007). Some consequences of counterbalancing excitation at and below threshold are regulating the duration of spike trains and interspike intervals and inducing oscillatory behavior. Concerning oscillatory behavior, striatal cholinergic interneurons show an intrinsically generated oscillatory spiking pattern with a frequency of $3-10 \mathrm{~Hz}$, which is about the firing rate 
Table 1. $I_{\text {sAHP }}$ contributes to the MAHP in striatal cholinergic interneurons

\begin{tabular}{|c|c|c|c|c|}
\hline & Control rats & 6-OHDA rats & Control rats + barium & 6-OHDA rats + barium \\
\hline \multicolumn{5}{|l|}{ First action potential in spike train } \\
\hline Threshold (mV) & $-41.1 \pm 1.6$ & $-44.2 \pm 1.9$ & $-42.8 \pm 1.4$ & $-46.5 \pm 1.8$ \\
\hline Max rising phase slope (mV/ms) & $218.0 \pm 13.8$ & $260.8 \pm 18.5$ & $220.9 \pm 9.0$ & $230.6 \pm 11.9$ \\
\hline Amplitude $(\mathrm{mV})^{a}$ & $79.4 \pm 3.0$ & $87.2 \pm 3.1$ & $87.8 \pm 2.0$ & $94.0 \pm 2.2$ \\
\hline Width at half amplitude (ms) ${ }^{b}$ & $0.45 \pm 0.01$ & $0.50 \pm 0.02$ & $0.61 \pm 0.02$ & $0.67 \pm 0.02$ \\
\hline mAHP amplitude $(\mathrm{mV})^{c}$ & $17.9 \pm 1.4$ & $12.8 \pm 1.5$ & $2.95 \pm 0.6$ & $7.40 \pm 1.1$ \\
\hline \multicolumn{5}{|c|}{ Last control action potential and time-matched action potential in 6-OHDA rats } \\
\hline Threshold (mV) & $-48.1 \pm 0.8$ & $-48.6 \pm 1.1$ & $-45.1 \pm 1.6$ & $-48.8 \pm 1.3$ \\
\hline Max rising phase slope $(\mathrm{mV} / \mathrm{ms})^{d}$ & $207.3 \pm 11.8$ & $197.5 \pm 13.6$ & $146.0 \pm 14.8$ & $166.5 \pm 12.2$ \\
\hline Amplitude (mV) & $87.0 \pm 1.9$ & $91.9 \pm 2.1$ & $80.0 \pm 3.8$ & $89.1 \pm 3.6$ \\
\hline Width at half amplitude (ms) & $1.10 \pm 0.08$ & $1.18 \pm 0.07$ & $2.31 \pm 0.39$ & $1.98 \pm 0.24$ \\
\hline mAHP amplitude $(\mathrm{mV})^{f}$ & $10.3 \pm 0.6$ & $6.5 \pm 0.6$ & $5.7 \pm 0.5$ & $4.9 \pm 0.4$ \\
\hline
\end{tabular}

Parameters are from spike trains evoked with $1 \mathrm{~s}, 230 \mathrm{pA}$ current pulses. Data are mean $\pm \mathrm{SEM}$ of $n=10$ control interneurons and $n=16$ interneurons from 6-0HDA rats. Amplitudes were measured from threshold. Statistical testing was performed with a two-way ANOVA with before and after barium being considered as a repeated measure.

${ }^{a}$ Significant lesion $(p=0.01)$ and barium effects $(p=0.0064)$ without interaction.

${ }^{b}$ Significant barium effect $(p<0.0001)$ without lesion effect and interaction.

'Significant barium effect $(p<0.0001)$ and interaction $(p=0.0003)$, NS lesion effect $(p=0.8)$. Bonferroni post hoc comparisons show more significant effects of barium in control $(p<0.001)$ than 6-0HDA rats $(p<0.05)$.

${ }^{d}$ Significant barium effect $(p=0.0002)$ without lesion effect and interaction. The increased excitability under barium may compromise recovery of the action current. Note that barium has no effect on the first spike rising phase slope.

${ }^{e}$ Significant barium effect $(p<0.0001)$ without lesion effect and interaction.

${ }^{f_{S}}$ Significant barium effect $(p<0.0001)$, lesion effect $(p=0.0052)$, and interaction $(p=0.0001)$. Bonferroni post hoc comparisons show a significant difference between groups before barium ( $\left.p<0.001\right)$, which becomes NS under barium $(p>0.05)$. Moreover, barium had a more significant effect in controls $(p<0.001)$ than $6-0$ HDA rats $(p<0.05)$.

that TANs show in vivo. Previous studies have identified the mechanism underlying this repetitive spiking pattern. Action potentials trigger calcium entry, resulting in activation of $I_{\mathrm{SK}}$, which induces an MAHP. In turn, hyperpolarizationactivated $I_{\mathrm{h}}$ and the persistent sodium current impel the cell again to threshold (Bennett et al., 2000; Maurice et al., 2004; Deng et al., 2007). According to this view, the interplay between $I_{\mathrm{SK}}$ and the abovementioned inward currents determines the firing rate during repetitive spiking. Our findings indicate that bariumsensitive $I_{\mathrm{sAHP}}$ is active at potentials at which the intrinsic pacemaker mechanism is at work and even at subthreshold potentials [see also Reynolds et al. (2004) and Ding et al. (2006)]. Therefore, the barium-sensitive component of $I_{\text {sAHP }}$ is not part of the pacemaker mechanism itself, but may moderate its pace. In physiological conditions, TANs are probably under continuous synaptic bombardment and $I_{\text {sAHP }}$ may additionally contribute to regulate tonic firing by counterbalancing synaptic excitation on a longer timescale. Thus, for comparable degrees of somatic excitation, the reduction of bariumsensitive $I_{\text {sAHP }}$ results in a higher preferred frequency of tonic firing in rats with nigrostriatal lesion.

In a natural context, striatal cholinergic interneurons should be under continuous synaptic influences, excitatory from the thalamus and to a lesser extent from the cerebral cortex (Lapper and Bolam, 1992; Thomas et al., 2000; Ding et al., 2010), and inhibitory through local circuitry driven by the cortex and thalamus (Suzuki et al., 2001; Sullivan et al., 2008). Afferent thalamic activity increases just before a pause

A
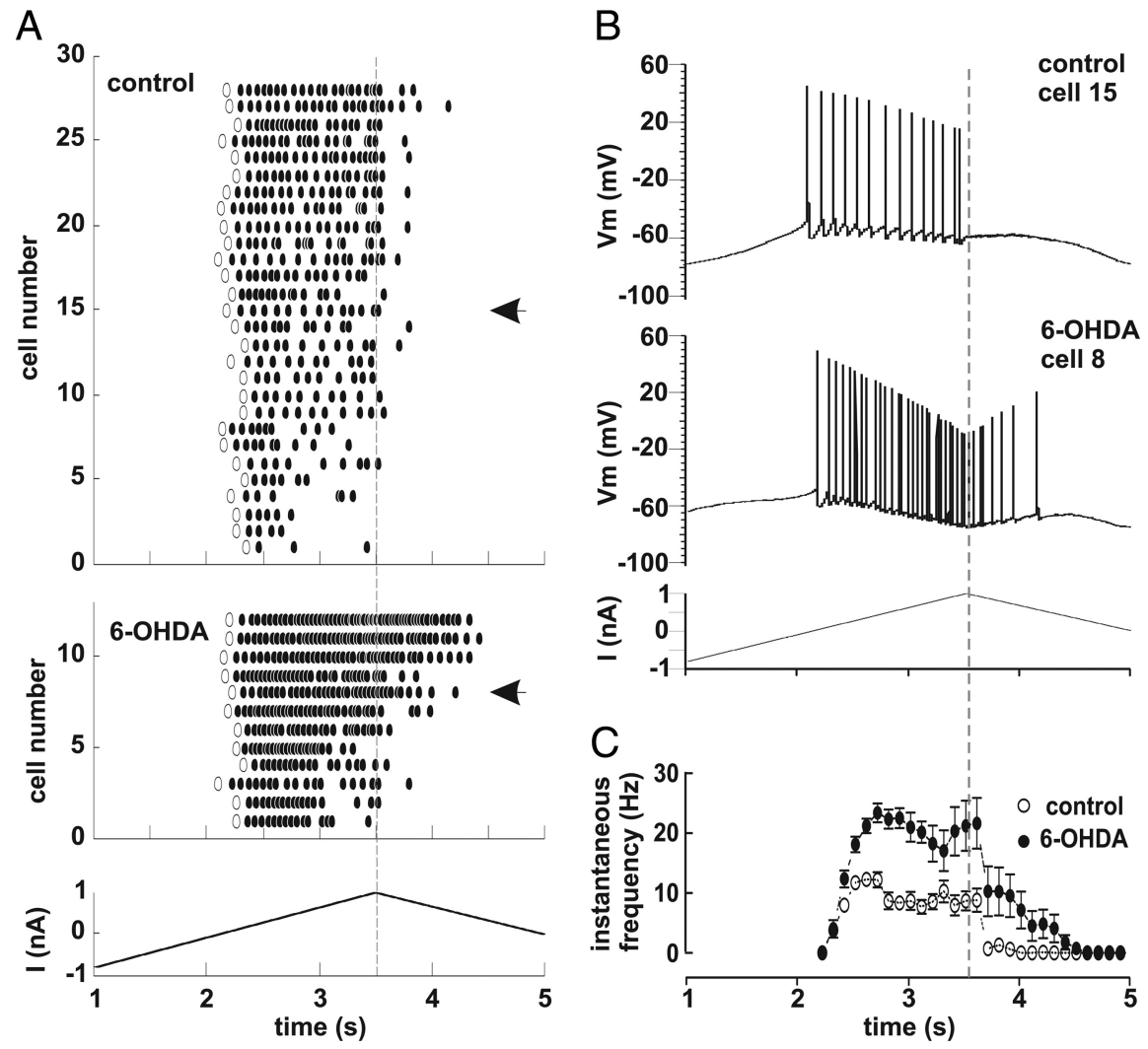

Figure 11. Increased preferred frequency of repetitive spiking and delayed transition to quiescence in rats with nigrostriatal lesion. $\boldsymbol{A}$, Raster plot showing the spiking response of striatal cholinergic interneurons in control (top) and 6-OHDA (bottom) rats to a depolarizing ramp followed by a hyperpolarizing ramp (depicted underneath). Each line is a different neuron. The first spike in each series is marked with an unfilled symbol. Arrows refer to the examples shown in $\boldsymbol{B}$. $\boldsymbol{C}$, Instantaneous frequency during the depolarizing ramp. The data were binned $(25 \mathrm{~ms})$ and averaged to give an estimate of the population change in firing rate as current changes during the ramp. Data are mean \pm SEM.

to a reward-related cue occurs. Moreover, inhibition of this thalamic activity by local administration of muscimol abolishes the pause response of TANs (Matsumoto et al., 2001). Coincident with the increase in thalamic activity, a brief discharge often precedes pauses in TANs (Kimura et al., 1984; Apicella et al., 1991; 
Aosaki et al., 1994; Matsumoto et al., 2001; Blazquez et al., 2002; Ravel et al., 2003; Morris et al., 2004). Finally, dopaminergic neurons fire a burst in coincidence with the pause in TANs during behavioral conditioning (Morris et al., 2004). In this context of afferent signals informing about outcomes and reward-related cues, several intrinsic mechanisms may contribute to the pause response. Currents mediating AHPs are candidates to underlie the pause (Reynolds et al., 2004; Wilson, 2005). According to one view, synaptic inhibition interacts with a hyperpolarizationactivated barium-sensitive outward current $\left(\mathrm{K}_{\mathrm{IR}}\right)$ to trigger a regenerative "all-or-none" hyperpolarization with a time course similar to the sAHP (Wilson, 2005). However, in vivo intracellular studies show that excitation induced by depolarizing current injection, but not hyperpolarizing current, causes an interruption of regular spiking in striatal cholinergic interneurons (Reynolds et al., 2004; see also Deng et al., 2007). Moreover, a recent slice study shows that thalamic stimulation induces a burst-pause response in striatal cholinergic interneurons regardless of $\mathrm{GABA}_{\mathrm{A}}$ receptor blockade, indicating that excitatory afferents do not need to engage local GABAergic circuitry to induce a pause (Ding et al., 2010). Similarly, Oswald et al. (2009) reported that cortical stimulation induces GABA-independent hyperpolarizing responses in striatal cholinergic interneurons. Although these findings do not rule out a contribution of inhibitory input to the pause in natural settings, they suggest that direct synaptic excitation of cholinergic interneurons may be sufficient to trigger a pause.

Depolarization may induce an intrinsic pause by different mechanisms. It may deactivate inward currents with slow kinetics that contribute to pacemaking, like $I_{\mathrm{h}}$, so that, when afferent excitation recedes, the membrane potential may fall below the level at which tonic firing is possible (Oswald et al., 2009). Alternatively, depolarization may recruit outward currents with slow kinetics like $I_{\mathrm{sAHP}}$. A simplified view of how $I_{\mathrm{sAHP}}$ contributes to a pause has led to equaling the pause with sAHP. However, natural pauses are briefer $(\sim 200 \mathrm{~ms})$ than sAHP (several hundred milliseconds to seconds). In fact, as TANs fire a few times per second, changes in the timing of a few spikes in every trial are what, after adding tens of trials, is seen as an excitation followed by a pause in the perievent histograms that usually illustrate neuronal responses in behavioral studies [for examples, see Kimura et al. (1984), their Fig. 1, or Morris et al. (2004), their Fig. 4]. We favor a view where barium-sensitive $I_{\mathrm{sAHP}}$ is active in a more graded and tonic fashion, depending on the previous history of the membrane potential. Because of its slow time course, a sudden reduction in afferent excitatory activity may leave its influence unbalanced, resulting in a pause. This is probably why in our ramp experiments most cholinergic interneurons abruptly stop firing when depolarizing current starts to ramp down (Fig. 11 A) despite the fact that the amount of current flowing into the cell body remains well above the threshold current defined during the ramp. Importantly, the excitatory thalamic input that drives the response of TANs to reward-related events shows a very specific temporal pattern, consisting in an increase in firing followed by a period of reduced activity and a delayed second wave of excitation (Matsumoto et al., 2001). Notably, the pause in TANs coincides very precisely with the interval of reduced firing in excitatory thalamic neurons (Matsumoto et al., 2001). Thus, the pause response, rather than reflecting a stereotyped response like the sAHP, may result from more subtle influences of $I_{\mathrm{sAHP}}$ and other currents on the ability of cholinergic interneurons to synchronize within the thalamostriatal network (Izhikevich, 2007). Recent studies show that the availability of slow currents like $I_{\mathrm{M}}$ and $I_{\mathrm{sAHP}}$ favors spike-time over spike-rate coding and promotes synchronization with excitatory input signals (Prescott et al., 2006, 2008; Prescott and Sejnowski, 2008). In this context, a reduction of $I_{\mathrm{sAHP}}$ may compromise the ability of striatal cholinergic interneurons to synchronize with excitatory inputs from the thalamus, leading to a degradation of TAN responses to rewardrelated events in parkinsonism.

How does the diminution of barium-sensitive $I_{\mathrm{sAHP}}$ relate to dopamine depletion? Ding et al. (2006) showed that dopamine depletion leads to a loss of muscarinic autoreceptor control in striatal cholinergic interneurons. Muscarinic autoreceptors inhibit cholinergic interneurons through a slow barium-sensitive potassium current (Calabresi et al., 1998), which resembles the $\mathrm{M}$-current that mediates muscarinic receptor actions in other cholinergic and noncholinergic neurons (Nicoll, 1988). Importantly, muscarinic agonists reduce the rate of pacemaking in striatal cholinergic interneurons, partly through effects on a bariumsensitive potassium current, and this effect is lost in an animal model of Parkinson's disease (Ding et al., 2006). Finally, cholinergic modulation of $I_{\mathrm{M}}$ and $I_{\mathrm{sAHP}}$ has dramatic effects on spike-frequency adaptation and the types of bifurcations (transitions between spiking and quiescent states) a neuron can pass through (Stiefel et al., 2009). In turn, a higher preferred frequency of firing and an altered encoding of salient sensory events in cholinergic interneurons may translate into abnormal synchronization of striatal cell assemblies (Carrillo-Reid et al., 2009) and a biased gating of cortical signals through the indirect basal ganglia pathway (Ding et al., 2010), contributing to the origin of Parkinson's disease symptoms.

\section{References}

Adams PR, Brown DA, Constanti A (1982) Pharmacological inhibition of the M-current. J Physiol 332:223-362.

Albin RL, Young AB, Penney JB (1989) The functional anatomy of basal ganglia disorders. Trends Neurosci 12:366-375.

Aosaki T, Graybiel AM, Kimura M (1994) Effect of the nigrostriatal dopamine system on acquired neural responses in the striatum of behaving monkeys. Science 265:412-415.

Apicella P, Scarnati E, Schultz W (1991) Tonically discharging neurons of monkey striatum respond to preparatory and rewarding stimuli. Exp Brain Res 84:672-675.

Barbeau A (1962) The pathogenesis of Parkinson's disease: a new hypothesis. Can Med Assoc J 87:802-807.

Bean BP (2007) The action potential in mammalian central neurons. Nat Rev Neurosci 8:451-465.

Bennett BD, Wilson CJ (1999) Spontaneous activity of neostriatal cholinergic interneurons in vitro. J Neurosci 19:5586-5596.

Bennett BD, Callaway JC, Wilson CJ (2000) Intrinsic membrane properties underlying spontaneous tonic firing in neostriatal cholinergic interneurons. J Neurosci 20:8493-8503.

Bergman H, Feingold A, Nini A, Raz A, Slovin H, Abeles M, Vaadia E (1998) Physiological aspects of information processing in the basal ganglia of normal and parkinsonian primates. Trends Neurosci 21:32-38.

Blazquez PM, Fujii N, Kojima J, Graybiel AM (2002) A network representation of response probability in the striatum. Neuron 33:973-982.

Calabresi P, Centonze D, Pisani A, Sancesario G, North RA, Bernardi G (1998) Muscarinic IPSPs in rat striatal cholinergic interneurones. J Physiol 510:421-427.

Carrillo-Reid L, Tecuapetla F, Ibáñez-Sandoval O, Hernández-Cruz A, Galarraga E, Bargas J (2009) Activation of the cholinergic system endows compositional properties to striatal cell assemblies. J Neurophysiol 101:737-749.

Cenci MA, Whishaw IQ, Schallert T (2002) Animal models of neurological deficits: how relevant is the rat? Nat Rev Neurosci 3:574-579.

Cooper EC, Harrington E, Jan YN, Jan LY (2001) M channel KCNQ2 subunits are localized to key sites for control of neuronal network oscillations and synchronization in mouse brain. J Neurosci 21:9529-9540.

DeBoer P, Heeringa MJ, Abercrombie ED (1996) Spontaneous release of 
acetylcholine in striatum is preferentially regulated by inhibitory dopamine D2 receptors. Eur J Pharmacol 317:257-262.

Deng P, Zhang Y, Xu ZC (2007) Involvement of I(h) in dopamine modulation of tonic firing in striatal cholinergic interneurons. J Neurosci $27: 3148-3156$.

Ding J, Guzman JN, Tkatch T, Chen S, Goldberg JA, Ebert PJ, Levitt P, Wilson CJ, Hamm HE, Surmeier DJ (2006) RGS4-dependent attenuation of M4 autoreceptor function in striatal cholinergic interneurons following dopamine depletion. Nat Neurosci 9:832-842.

Ding JB, Guzman JN, Peterson JD, Goldberg JA, Surmeier DJ (2010) Thalamic gating of corticostriatal signaling by cholinergic interneurons. Neuron 67:294-307.

Faber ES, Sah P (2005) Independent roles of calcium and voltage-dependent potassium currents in controlling spike frequency adaptation in lateral amygdala pyramidal neurons. Eur J Neurosci 22:1627-1635.

Fino E, Glowinski J, Venance L (2007) Effects of acute dopamine depletion on the electrophysiological properties of striatal neurons. Neurosci Res 58:305-316.

Goldberg JA, Wilson CJ (2005) Control of spontaneous firing patterns by the selective coupling of calcium currents to calcium-activated potassium currents in striatal cholinergic interneurons. J Neurosci 25:10230-10238.

Goldberg JA, Teagarden MA, Foehring RC, Wilson CJ (2009) Nonequilibrium calcium dynamics regulate the autonomous firing pattern of rat striatal cholinergic interneurons. J Neurosci 29:8396-8407.

Hines ML, Carnevale NT (1997) The NEURON simulation environment. Neural Comput 9:1179-1209.

Izhikevich EM (2007) Dynamical systems in neuroscience: the geometry of excitability and bursting. Cambridge, MA: MIT Press.

Jiang ZG, North RA (1991) Membrane properties and synaptic responses of rat striatal neurones in vitro. J Physiol 443:533-553.

Khaliq ZM, Gouwens NW, Raman IM (2003) The contribution of resurgent sodium current to high-frequency firing in Purkinje neurons: an experimental and modeling study. J Neurosci 23:4899-4912.

Kimura M, Rajkowski J, Evarts E (1984) Tonically discharging putamen neurons exhibit set-dependent responses. Proc Natl Acad Sci U S A 81:4998-5001.

Lapper SR, Bolam JP (1992) Input from the frontal cortex and the parafascicular nucleus to cholinergic interneurons in the dorsal striatum of the rat. Neuroscience 51:533-545.

Lazarewicz MT, Migliore M, Ascoli GA (2002) A new bursting model of CA3 pyramidal cell physiology suggests multiple locations for spike initiation. Biosystems 67:129-137.

Madison DV, Nicoll RA (1984) Control of the repetitive discharge of rat CA 1 pyramidal neurones in vitro. J Physiol 354:319-331.

Matsumoto N, Minamimoto T, Graybiel AM, Kimura M (2001) Neurons in the thalamic CM-Pf complex supply striatal neurons with information about behaviorally significant sensory events. J Neurophysiol 85:960-976.

Maurice N, Mercer J, Chan CS, Hernandez-Lopez S, Held J, Tkatch T, Surmeier DJ (2004) $\mathrm{D}_{2}$ dopamine receptor-mediated modulation of voltage-dependent $\mathrm{Na}^{+}$channels reduces autonomous activity in striatal cholinergic interneurons. J Neurosci 24:10289-10301.

Morris G, Arkadir D, Nevet A, Vaadia E, Bergman H (2004) Coincident but distinct messages of midbrain dopamine and striatal tonically active neurons. Neuron 43:133-143.

Nakagawa M, Ohgoh M, Nishizawa Y, Ogura H (2004) Dopaminergic agonists and muscarinic antagonists improve lateralization in hemiparkinsonian rats in a novel exploratory Y-maze. J Pharmacol Exp Ther 309:737-744.

Nicoll RA (1988) The coupling of neurotransmitter receptors to ion channels in the brain. Science 241:545-551.

Oswald MJ, Oorschot DE, Schulz JM, Lipski J, Reynolds JN (2009) IH current generates the afterhyperpolarisation following activation of subthreshold cortical synaptic inputs to striatal cholinergic interneurons. J Physiol 587:5879-5897.

Paxinos G, Watson C (1997) The rat brain in stereotaxic coordinates. San Diego: Academic.

Pisani A, Bernardi G, Ding J, Surmeier DJ (2007) Re-emergence of striatal cholinergic interneurons in movement disorders. Trends Neurosci 30:545-553.
Prescott SA, Sejnowski TJ (2008) Spike-rate coding and spike-time coding are affected oppositely by different adaptation mechanisms. J Neurosci 28:13649-13661.

Prescott SA, Ratté S, De Koninck Y, Sejnowski TJ (2006) Nonlinear interaction between shunting and adaptation controls a switch between integration and coincidence detection in pyramidal neurons. J Neurosci 26:9084-9097.

Prescott SA, Ratté S, De Koninck Y, Sejnowski TJ (2008) Pyramidal neurons switch from integrators in vitro to resonators under in vivo-like conditions. J Neurophysiol 100:3030-3042.

Ravel S, Legallet E, Apicella P (2003) Responses of tonically active neurons in the monkey striatum discriminate between motivationally opposing stimuli. J Neurosci 23:8489-8497.

Reynolds JN, Hyland BI, Wickens JR (2004) Modulation of an afterhyperpolarization by the substantia nigra induces pauses in the tonic firing of striatal cholinergic interneurons. J Neurosci 24:9870-9877.

Schultz W (2007) Multiple dopamine functions at different time courses. Annu Rev Neurosci 30:259-288.

Song WJ, Tkatch T, Baranauskas G, Ichinohe N, Kitai ST, Surmeier DJ (1998) Somatodendritic depolarization-activated potassium currents in rat neostriatal cholinergic interneurons are predominantly of the A type and attributable to coexpression of Kv4.2 and Kv4.1 subunits. J Neurosci 18:3124-3137.

Stiefel KM, Gutkin BS, Sejnowski TJ (2009) The effects of cholinergic neuromodulation on neuronal phase-response curves of modeled cortical neurons. J Comput Neurosci 26:289-301.

Stocker M (2004) Ca(2+)-activated K+ channels: molecular determinants and function of the SK family. Nat Rev Neurosci 5:758-770.

Storm JF (1990) Potassium currents in hippocampal pyramidal cells. Prog Brain Res 83:161-187.

Sullivan MA, Chen H, Morikawa H (2008) Recurrent inhibitory network among striatal cholinergic interneurons. J Neurosci 28:8682-8689.

Suzuki T, Miura M, Nishimura K, Aosaki T (2001) Dopamine-dependent synaptic plasticity in the striatal cholinergic interneurons. J Neurosci 21:6492-6501.

Thomas TM, Smith Y, Levey AI, Hersch SM (2000) Cortical inputs to m2immunoreactive striatal interneurons in rat and monkey. Synapse 37:252-361.

Thurley K, Senn W, Lüscher HR (2008) Dopamine increases the gain of the input-output response of rat prefrontal pyramidal neurons. J Neurophysiol 99:2985-2997.

Tseng KY, Kasanetz F, Kargieman L, Riquelme LA, Murer MG (2001) Cortical slow oscillatory activity is reflected in the membrane potential and spike trains of striatal neurons in rats with chronic nigrostriatal lesions. J Neurosci 21:6430-6439.

Tzingounis AV, Nicoll RA (2008) Contribution of KCNQ2 and KCNQ3 to the medium and slow afterhyperpolarization currents. Proc Natl Acad Sci U S A 105:19974-19979.

Wang HS, McKinnon D (1995) Potassium currents in rat prevertebral and paravertebral sympathetic neurones: control of firing properties. J Physiol 485:319-335.

Wang J, Chen S, Siegelbaum SA (2001) Regulation of hyperpolarizationactivated $\mathrm{HCN}$ channel gating and cAMP modulation due to interactions of $\mathrm{COOH}$ terminus and core transmembrane regions. J Gen Physiol 118:237-250.

Wilson CJ (2005) The mechanism of intrinsic amplification of hyperpolarizations and spontaneous bursting in striatal cholinergic interneurons. Neuron 45:575-585.

Wilson CJ, Goldberg JA (2006) Origin of the slow afterhyperpolarization and slow rhythmic bursting in striatal cholinergic interneurons. J Neurophysiol 95:196-204.

Yan Z, Surmeier DJ (1996) Muscarinic (m2/m4) receptors reduce N- and P-type $\mathrm{Ca}^{2+}$ currents in rat neostriatal cholinergic interneurons through a fast, membrane-delimited, G-protein pathway. J Neurosci 16:2592-2604.

Zhang ZW, Arsenault D (2005) Gain modulation by serotonin in pyramidal neurones of the rat prefrontal cortex. J Physiol 566:379-394.

Zold CL, Larramendy C, Riquelme LA, Murer MG (2007) Distinct changes in evoked and resting globus pallidus activity in early and late Parkinson's disease experimental models. Eur J Neurosci 26:1267-1279. 